\title{
Global Attractivity and Extinction for Lotka-Volterra systems with infinite delay and feedback controls
}

\author{
Teresa Faria *, a and Yoshiaki Muroya ${ }^{\mathrm{b}}$ \\ aDepartamento de Matemática and CMAF, Faculdade de Ciências, \\ Universidade de Lisboa, Campo Grande, 1749-016 Lisboa, Portugal \\ tfaria@ptmat.fc.ul.pt \\ ${ }^{\mathrm{b}}$ Department of Mathematics, Waseda University \\ 3-4-1 Ohkubo, Shinjuku-ku, Tokyo 169-8555, Japan \\ ymuroya@waseda.jp
}

\begin{abstract}
The paper deals with a multiple species Lotka-Volterra model with infinite distributed delays and feedback controls, for which we assume a weak form of diagonal dominance of the instantaneous negative intra-specific terms over the infinite delay effect in both the population variables and controls. General sufficient conditions for the existence and attractivity of a saturated equilibrium are established. When the saturated equilibrium is on the boundary of $\mathbb{R}_{+}^{n}$, sharper criteria for the extinction of all or part of the populations are given. While the literature usually treats the case of competitive systems only, here no restrictions on the signs of the intra- and inter-specific delayed terms are imposed. Moreover, our technique does not require the construction of Lyapunov functionals.
\end{abstract}

Keywords: Lotka-Volterra system, infinite delay, feedback control, saturated equilibrium, global attractivity, extinction.

2010 AMS Subject Classification: 34K20, 34K25, 92D25, 93B52.

* Corresponding author.Fax:+351 21795 4288; Tel: +351 217904929.

a The research was supported by Fundação para a Ciência e a Tecnologia (Portugal), PEst-OE/MAT/UI0209/2011.

b The research was supported by Scientific Research (c), No.24540219 of Japan Society for the Promotion of Science. 


\section{Introduction}

After several decades of intensive study and use of functional differential equations (FDEs) in population dynamics, it is now very well understood that the introduction of delays in differential equations leads in general to more realistic population models, and much more complex and rich dynamics. Nevertheless, delays are not harmless and often create instability and oscillations, unless they are either small or neutralized by instantaneous terms. When the delays are infinite, it is not clear how to surpass the effect of the infinite past of the system, so in order to obtain stability results some form of instantaneous dominance is expected. On the other hand, the consideration of FDEs with infinite delay is relevant to account for systems with "infinite memory", and goes back to the works of Volterra. In fact, for Lotka-Volterra systems or other general population models, whether the global stability may persist under large or even infinite delays without strictly dominating instantaneous negative feedbacks is a question that has attracted the interest of many researchers, and had partial positive answers, see e.g. Kuang [15], Xu et al. [26], Faria [4], also for further references.

Recently, the study of population models with delays and controls, in particular Lotka-Volterra models, has received some attention, see e.g. [3, 8, 16, 20, 21, 24, 27], and references therein. In this paper, we consider the following n-species Lotka-Volterra system with feedback controls and infinite delays:

$$
\left\{\begin{array}{l}
x_{i}^{\prime}(t)=x_{i}(t)\left(b_{i}-\mu_{i} x_{i}(t)-\sum_{j=1}^{n} a_{i j} \int_{0}^{\infty} K_{i j}(s) x_{j}(t-s) d s-c_{i} \int_{0}^{\infty} G_{i}(s) u_{i}(t-s) d s\right), \\
u_{i}^{\prime}(t)=-e_{i} u_{i}(t)+d_{i} x_{i}(t), \quad i=1,2, \ldots, n,
\end{array}\right.
$$

where: $\mu_{i}, c_{i}, d_{i}, e_{i}$ are positive constants, $b_{i}, a_{i j} \in \mathbb{R}$, and the kernels $K_{i j}, G_{i}:[0, \infty) \rightarrow[0, \infty)$ are $L^{1}$ functions, normalized so that

$$
\int_{0}^{\infty} K_{i j}(s) d s=1, \quad \int_{0}^{\infty} G_{i}(s) d s=1
$$

for $i, j=1,2, \ldots, n$. Without loss of generality, we assume that for all $i$ the linear operators defined by $L_{i i}(\varphi)=\int_{0}^{\infty} K_{i i}(s) \varphi(-s) d s$, for bounded continuous functions $\varphi:(-\infty, 0] \rightarrow \mathbb{R}$, are non-atomic at zero, which amounts to have $K_{i i}(0)=K_{i i}\left(0^{+}\right)$.

In biological terms, $x_{i}(t)$ denotes the density of the population $i$ with Malthusian growth rate $b_{i}$ and instantaneous self-limitation coefficient $\mu_{i}>0$, and $a_{i i}$ and $a_{i j}(i \neq j)$ are respectively the intra- and inter-specific delayed acting coefficients; $u_{i}(t)$ denotes a feedback control variable, $i, j=1,2, \ldots, n$. Due to the biological interpretation of model (1.1), we are only interested in positive (or non-negative) solutions. We therefore consider solutions of system (1.1) with admissible initial conditions, i.e.,

$$
x_{i}(\theta)=\varphi_{i}(\theta) \geq 0, u_{i}(\theta)=\psi_{i}(\theta) \geq 0, \theta \in(-\infty, 0), \quad \varphi_{i}(0)>0, \psi_{i}(0)>0,
$$

with $\varphi_{i}, \psi_{i}$ bounded continuous functions on $(-\infty, 0], i=1,2, \ldots, n$. 
In order to have an effective feedback control, it is natural to impose that each $G_{i}(1 \leq i \leq n)$ satisfies

$$
\int_{0}^{\infty} G_{i}(s) \varphi(-s) d s>0
$$

for any positive, bounded, continuous function $\varphi$ defined on $(-\infty, 0]$ with $\varphi(0)>0$. In particular, (1.3) holds if $G_{i}$ is continuous at zero with $G_{i}(0)>0$, or if $G_{i}$ has a jump discontinuity at 0 with $G_{i}(0)-G_{i}\left(0^{+}\right)>0$.

For simplicity of exposition, we consider systems (1.1), but our study applies to more general systems of the form

$$
\left\{\begin{array}{l}
x_{i}^{\prime}(t)=x_{i}(t)\left(b_{i}-\mu_{i} x_{i}(t)-\sum_{j=1}^{n} a_{i j} \int_{0}^{\infty} x_{j}(t-s) d \eta_{i j}(s)-c_{i} \int_{0}^{\infty} u_{i}(t-s) d \nu_{i}(s)\right) \\
u_{i}^{\prime}(t)=-e_{i} u_{i}(t)+d_{i} x_{i}(t), \quad i=1,2, \ldots, n
\end{array}\right.
$$

where all the coefficients are as in $(1.1)$, and $\eta_{i j}, \nu_{i}:[0, \infty) \rightarrow \mathbb{R}$ are bounded variation functions which are supposed to be normalized so that their total variation is 1 , and $\nu_{i}$ are non-decreasing on $[0, \infty)$. Note that in $(1.1)$ we supposed that $K_{i j}(t) \geq 0$ on $[0, \infty)$, but the above scenario does not impose this restriction. Some of our general results however require that the kernels $K_{i j}$ in (1.1) are non-negative, or that the functions $\eta_{i j}$ in (1.4) are non-decreasing, although they can be easily adapted to deal with systems without such constraints.

Our study was strongly motivated by some previous works of the present authors. The uncontrolled Lotka-Volterra system with infinite distributed delays was studied by Faria [4], and questions of partial survival and extinction of species in non-autonomous delayed Lotka-Volterra systems were addressed by Muroya in [19], see also [18]. The works of Gopalsamy and Weng [8] and Li et al. [16], where special cases of two-dimensional competitive Lotka-Volterra systems with controls and no diagonal delays were studied, were an important source of inspiration for the present paper. Here, the investigation refers to controlled Lotka-Volterra models of any dimension $n$. While the literature usually only deals with the case of competitive systems (i.e., systems with $a_{i j} \geq 0$ for $j \neq i$ ) with $b_{i}>0$, here no restrictions on the signs of $a_{i j}$ and $b_{i}$ will be imposed. Moreover, infinite delays are incorporated in the controls terms, see also [21] for a competitive model. Another novelty is that our method does not require the construction of a specific Lyapunov functional.

Clearly, the introduction of controls in a delayed Lotka-Volterra system might change the existence, position, and stability of equilibria. The main goal of the present paper is to address the global asymptotic dynamics of solutions to (1.1)-(1.2), in what concerns establishing sufficient conditions for the existence and attractivity of a saturated equilibrium (see $[14,15]$ and Section 3 for a definition) not necessarily positive. As in previous works $[4,8,16,21]$, we assume that system (1.1) satisfies some form of diagonal dominance of the instantaneous negative terms $\mu_{i} x_{i}(t)$ over the infinite delay terms, involving both the population variables and the controls, so that the usual instability caused by the introduction of the delays is canceled. For some of our stability results, another prerequisite is that the uncontrolled Lotka-Volterra, system $(1.1)$ with $c_{i}=0(1 \leq i \leq n)$, 
possesses already a globally attractive saturated equilibrium. These assumptions, although they seem restrictive, are quite natural; moreover, here the main goal is to use the controls to change the position of the saturated equilibrium keeping its stability, as emphasised by some examples. For a biological interpretation of the use of controls, see e.g. [8, 24, 27], also for additional references.

We now describe briefly the contents of the paper. From a theoretical perspective, dealing with FDEs with infinite delays requires a careful choice of a suitable Banach phase space (usually called a fading memory space), in order to recover classical results of well-posedness of the initial value problem, existence and uniqueness of solutions, continuation of solutions, etc. For this reason, in Section 2 we set some basic notation for FDEs with infinite delays, and insert system (1.1) into such a framework. In Section 3, after studying the existence of a unique saturated equilibrium $\left(x^{*}, u^{*}\right)$ and the boundedness of positive solutions to (1.1), Theorems 3.2 provides a general criterion for the global attractivity of $\left(x^{*}, u^{*}\right)$. Also, a sufficient condition for the dissipativeness of (1.1) is given. In Section 4, sharper criteria are established for the global attractivity of a saturated equilibrium $\left(x^{*}, u^{*}\right)$ which is not strictly positive. In this situation, this means the extinction of all or part of the populations. Our results turn out to be particularly powerful for predator-prey models. We also emphasize that, for the uncontrolled system, we derive better results for partial (or total) extinction than the ones in [4]. Our techniques also allow to obtain a perturbation result for nonautonomous Lotka-Volterra systems with a limiting model of the form (1.1) or (1.4), as $t \rightarrow \infty$. Section 5 is devoted to the particular case of a 2-dimensional Lotka-Volterra system. In Sections 4 and 5, some examples illustrate our results.

\section{An abstract formulation}

Since system (1.1) has unbounded delays, we must carefully formulate the problem by defining an appropriate Banach phase space where the problem is well-posed.

Let $g$ be a function satisfying the following properties:

(g1) $g:(-\infty, 0] \rightarrow[1, \infty)$ is a non-increasing continuous function and $g(0)=1$;

(g2) $\lim _{u \rightarrow 0^{-}} \frac{g(s+u)}{g(s)}=1$ uniformly on $(-\infty, 0]$;

(g3) $g(s) \rightarrow \infty$ as $s \rightarrow-\infty$.

For $n \in \mathbf{N}$, define the Banach space $U C_{g}=U C_{g}\left(\mathbb{R}^{n}\right)$,

$$
U C_{g}:=\left\{\phi \in C\left((-\infty, 0] ; \mathbb{R}^{n}\right): \sup _{s \leq 0} \frac{|\phi(s)|}{g(s)}<\infty, \frac{\phi(s)}{g(s)} \text { is uniformly continuous on }(-\infty, 0]\right\}
$$

with the norm

$$
\|\phi\|_{g}=\sup _{s \leq 0} \frac{|\phi(s)|}{g(s)},
$$

where $|\cdot|$ is a chosen norm in $\mathbb{R}^{n}$. Consider also the space $B C=B C\left(\mathbb{R}^{n}\right)$ of bounded continuous functions $\phi:(-\infty, 0] \rightarrow \mathbb{R}^{n}$. It is clear that $B C \subset U C_{g}$, with $\|\phi\|_{g} \leq\|\phi\|_{\infty}$ for $\phi \in B C$ and $\|\cdot\|_{\infty}$ 
the supremum norm in $B C$. Here, $B C$ will be considered as a subspace of $U C_{g}$, so $B C$ is endowed with the norm of $U C_{g}$.

The space $U C_{g}$ is an admissible phase space for $n$-dimensional FDEs with infinite delay (cf. $[12,13])$ written in the abstract form

$$
\dot{x}(t)=f\left(t, x_{t}\right)
$$

where $f: D \subset \mathbb{R} \times U C_{g} \rightarrow \mathbb{R}^{n}$ is continuous and, as usual, segments of solutions in the phase space $U C_{g}$ are denoted by $x_{t}, x_{t}(s)=x(t+s), s \leq 0$, with components $x_{t, i}$. Therefore, the standard results on existence and uniqueness of solutions for the Cauchy problem $\dot{x}(t)=f\left(t, x_{t}\right), x_{0}=\varphi$ hold when $f$ is regular enough and $\varphi \in B C$. Moreover, since $U C_{g}$ is a fading memory space, bounded positive orbits are precompact in $U C_{g}[9]$.

We now set an appropriate formulation for problem (1.1)-(1.2). From [10] and [6, Lemma 4.1], for any $\delta>0$ there is a continuous function $g$ satisfying (g1)-(g3) and such that

$$
\int_{0}^{\infty} g(-s) K_{i j}(s) d s<1+\delta, \int_{0}^{\infty} g(-s) G_{i}(s) d s<1+\delta, \quad i, j=1, \ldots, n .
$$

When dealing with systems (1.4), where the more general linearities are given by bounded variation functions $\eta_{i j}(s), \nu_{i}(s)$ with total variation 1 and $\nu_{i}(s)$ non-decreasing, the above formulas $(2.2)$ should be replaced by

$$
\int_{0}^{\infty} g(-s) d\left|\eta_{i j}(s)\right|<1+\delta, \int_{0}^{\infty} g(-s) d \nu_{i}(s)<1+\delta, \quad i, j=1, \ldots, n .
$$

Whenever an abstract setting is required, in what follows we shall always assume that (1.1) takes the abstract form (2.1) in the phase space $U C_{g}=U C_{g}\left(\mathbb{R}^{2 n}\right)$, for some fixed $\delta>0$ and function $g$ satisfying (g1)-(g3) and (2.2), and consider solutions with initial conditions

$$
x_{0}=\varphi, \quad u(0)=\psi
$$

where $(\varphi, \psi) \in B C\left(\mathbb{R}^{2 n}\right)$. System (1.1) has a unique solution $(x(t), u(t))=(x(t ; \varphi, \psi), u(t ; \varphi, \psi))$ satisfying (2.4). Moreover, since only positive or non-negative solutions of (1.1) are biologically meaningful, we restrict our framework to positive or non-negative initial conditions. A vector $x \in \mathbb{R}^{n}$ is said to be positive, or non-negative, if all its components are positive, or non-negative, respectively, and we write $x>0, x \geq 0$, respectively. We define and denote in a similar way positive and non-negative functions in $B C$, and positive and non-negative matrices as well. As usual, we use the notation $\mathbb{R}_{+}^{n}=\left\{x \in \mathbb{R}^{n}: x \geq 0\right\}$. In the space $U C_{g}$, a vector $c$ is identified with the constant function $\psi(s)=c$ for $s \leq 0$.

Consider the positive cone $B C^{+}=B C^{+}\left(\mathbb{R}^{2 n}\right)=\{(\varphi, \psi) \in B C: \varphi(s), \psi(s) \geq 0$ for all $s \leq 0\}$. As set of admissible initial conditions for (1.1), we take the subset $B C_{0}^{+}$of $B C^{+}, B C_{0}^{+}=$ $\left\{(\varphi, \psi) \in B C^{+}: \varphi(0)>0, \psi(0)>0\right\}$. It is easy to see that all the coordinates of solutions with 
initial conditions in $B C^{+}$, respectively $B C_{0}^{+}$, remain nonnegative, respectively positive, for all $t \geq 0$ whenever they are defined.

In the sequel, we shall consider norms $|\cdot|_{d}$ in $\mathbb{R}^{N}(N=n$ or $N=2 n)$ given by $\left|\left(x_{1}, \ldots, x_{N}\right)\right|_{d}=$ $\max _{1 \leq i \leq N} d_{i}\left|x_{i}\right|$, for some $d=\left(d_{1}, \ldots, d_{N}\right)>0$. For such norms in $\mathbb{R}^{N}$, in order to be more explicit, we denote the norm in $U C_{g}$ by $\|\cdot\|_{g, d}$,

$$
\|\psi\|_{g, d}=\sup _{s \leq 0} \frac{|\psi(s)|_{d}}{g(s)} .
$$

\section{Existence and global attractivity of a saturated equilibrium}

In the absence of controls, the Lotka-Volterra system reads as

$$
x_{i}^{\prime}(t)=x_{i}(t)\left(b_{i}-\mu_{i} x_{i}(t)-\sum_{j=1}^{n} a_{i j} \int_{0}^{\infty} K_{i j}(s) x_{j}(t-s) d s\right),
$$

for which

$$
M_{0}=N+A, \quad \text { where } \quad N=\operatorname{diag}\left(\mu_{1}, \ldots, \mu_{n}\right), \quad A=\left[a_{i j}\right],
$$

is designated as the interaction community matrix. As for ordinary differential equations (ODEs), the algebraic properties of $M_{0}$ determine many features of the asymptotic behaviour of solutions to (3.1) (cf. e.g. [4, 5, 14]). Clearly, the introduction of controls might change the dynamics of (3.1). Here, the main aim is to use the controls to change the position of a globally attractive equilibrium, and give general criteria for its attractivity.

For (1.1), we define the controlled community matrix as

$$
M=N+A+C, \quad \text { where } \quad C=\operatorname{diag}\left(\frac{c_{1} d_{1}}{e_{1}}, \ldots, \frac{c_{n} d_{n}}{e_{n}}\right)
$$

We also consider the matrices

$$
\hat{M}_{0}=N-|A|, \quad \hat{M}=N-|A|-C, \quad \text { where } \quad|A|=\left[\left|a_{i j}\right|\right] .
$$

Note that $\left(x^{*}, u^{*}\right) \in \mathbb{R}^{n} \times \mathbb{R}^{n}$ is an equilibrium of (1.1) if and only if

$$
x_{i}^{*}=0 \text { or }\left(M x^{*}\right)_{i}=b_{i}, \quad \text { and } \quad u_{i}^{*}=\frac{d_{i}}{e_{i}} x_{i}^{*}, \quad i=1, \ldots, n .
$$

Throughout the paper, we shall use the definition of a saturated equilibrium.

Definition 3.1. Let $\left(x^{*}, u^{*}\right)=\left(x_{1}^{*}, \ldots, x_{n}^{*}, u_{1}^{*}, \ldots, u_{n}^{*}\right)$ be an equilibrium of (1.1). We say that $\left(x^{*}, u^{*}\right)$ is a saturated equilibrium if $\left(x^{*}, u^{*}\right)$ is non-negative and

$$
\left(M x^{*}\right)_{i} \geq b_{i} \quad \text { whenever } \quad x_{i}^{*}=0, \quad i=1, \ldots, n .
$$


Remark 3.1. We observe that if $\left(x^{*}, u^{*}\right) \geq 0$ is an equilibrium of (1.1) on the border of the positive cone $\mathbb{R}_{+}^{n} \times \mathbb{R}_{+}^{n}$, i.e., $x_{i}^{*}=u_{i}^{*}=0$ for some $i$, and $\left(x^{*}, u^{*}\right)$ is not saturated, then $\left(x^{*}, u^{*}\right)$ is unstable. In fact, system $(1.1)$ and the ODE system in $\mathbb{R}_{+}^{n} \times \mathbb{R}_{+}^{n}$

$$
\left\{\begin{array}{l}
x_{i}^{\prime}(t)=x_{i}(t)\left(b_{i}-\mu_{i} x_{i}(t)-\sum_{j=1}^{n} a_{i j} x_{j}(t)-c_{i} u_{i}(t)\right), \\
u_{i}^{\prime}(t)=-e_{i} u_{i}(t)+d_{i} x_{i}(t), \quad i=1,2, \ldots, n,
\end{array}\right.
$$

share the same equilibria. Since $\mathbb{R}_{+}^{n} \times \mathbb{R}_{+}^{n}$ is forward invariant for $(3.5)$, if $\left(x^{*}, u^{*}\right) \geq 0$ is an equilibrium of $(3.5)$ and $\left(x^{*}, u^{*}\right)$ is not saturated, then $\left(x^{*}, u^{*}\right)$ is unstable, since the characteristic equation for the linearised equation about $\left(x^{*}, u^{*}\right)$ has an eigenvalue with positive real part (cf. e.g. [14]).

When analysing (1.1), our concepts of attractivity and stability always refer to the set of admissible solutions, i.e., to solutions $(x(t), u(t))=(x(t ; \varphi, \psi), u(t ; \varphi, \psi))$ with $(\varphi, \psi)$ in the set of admissible initial conditions. In particular, an equilibrium $\left(x^{*}, u^{*}\right)$ of $(1.1)$ is globally attractive if all solutions $(x(t), u(t))$ of $(1.1)$ with initial conditions $\left(x_{0}, u_{0}\right)=(\varphi, \psi) \in B C_{0}^{+}$, satisfy $\lim _{t \rightarrow \infty} x(t)=$ $x^{*}, \lim _{t \rightarrow \infty} u(t)=u^{*}$; and it is globally asymptotically stable (GAS) if it is stable and globally attractive.

We recall some concepts from matrix theory which will be used in the next sections.

Definition 3.2. Let $B=\left[b_{i j}\right]$ be an $n \times n$ matrix. We say that $B$ is an M-matrix (respectively non-singular M-matrix) if $b_{i j} \leq 0$ for $i \neq j$ and all its eigenvalues have non-negative (respectively positive) real parts. The matrix $B$ is said to be a $\mathbf{P}$-matrix if all its principal minors are positive.

Remark 3.2. It is well-known that there are several equivalent ways of defining M-matrices, non-singular M-matrices and P-matrices; in [7], these matrices are also designated by matrices of classes $K_{0}, K$ and $P$, respectively. See $[1,7,14]$ for further properties of these matrices. In particular, we recall that a square matrix with non-positive off-diagonal entries is an M-matrix (respectively, a non-singular M-matrix) if and only if all its principal minors are non-negative (respectively, positive); so any non-singular M-matrix is a P-matrix. A related concept is the notation of a Volterra-Lyapunov stable (VL-stable for short) matrix, i.e., an $n \times n$ matrix $B=\left[b_{i j}\right]$ for which there exists a positive vector $d=\left(d_{1}, \ldots, d_{n}\right)$ such that $\sum_{i, j=1}^{n} x_{i} d_{i} b_{i j} x_{j}<0$ for all $x=$ $\left(x_{1}, \ldots, x_{n}\right) \neq 0$. If $-B$ is VL-stable then $B$ is also a $\mathrm{P}$-matrix; the converse is true for the particular case of a $2 \times 2$ matrix, but not for higher dimensions. For Lotka-Volterra ODE systems of the form $x_{i}^{\prime}=x_{i}\left[b_{i}-\sum_{j=1}^{n} a_{i j} x_{j}\right], 1 \leq i \leq n$, it is known that if $-\left[a_{i j}\right]$ is VL-stable, then there is one globally stable saturated equilibrium [14, p. 199].

Consider both the original and the controlled community matrices $M_{0}, M$, as well as the matrices $\hat{M}_{0}, \hat{M}$, cf. (3.2)-(3.4). For the uncontrolled system (3.1), it was shown in [4, Corollary 4.1] that if $\hat{M}_{0}$ is a non-singular M-matrix, then there is a unique saturated equilibrium of (3.1), which is a global attractor of all solutions with initial conditions $x_{0}=\varphi \in B C_{0}^{+}\left(\mathbb{R}^{n}\right)$. The idea 
now is to prove a similar result for system (1.1). We start by studying the existence of a saturated equilibrium and the boundedness of solutions to (1.1).

Theorem 3.1. Assume that $M$ is a $P$-matrix, where $M$ is the controlled community matrix in (3.3). Then, there is a unique saturated equilibrium $\left(x^{*}, u^{*}\right)$ of (1.1).

Proof. If $M$ is a P-matrix, then for each vector $b=\left(b_{1}, \ldots, b_{n}\right) \in \mathbb{R}^{n}$ there is a unique nonnegative vector $x^{*}$ such that $M x^{*} \geq b$ and $\left(M x^{*}\right)_{i}=b_{i}$ if $x_{i}^{*}>0\left[1\right.$, p. 274]. With $u^{*}=\left(u_{1}^{*}, \ldots, u_{n}^{*}\right)$ where $u_{i}^{*}=\frac{d_{i}}{e_{i}} x_{i}^{*}$, this means that $\left(x^{*}, u^{*}\right)$ is the unique saturated equilibrium of (1.1).

If all coefficients in (1.1) are positive, then clearly all positive solutions are bounded, since the inequalities $x_{i}^{\prime}(t) \leq x_{i}(t)\left(b_{i}-\mu_{i} x_{i}(t)\right)$ hold, and positive solutions of the logistic ODEs $y^{\prime}(t)=$ $y(t)\left(b_{i}-\mu_{i} y(t)\right)$ are bounded. This is not however the case if we allow some of the coefficients $a_{i j}$ to be negative, unless further constraints on $M_{0}$ are imposed.

Lemma 3.1. Assume that the matrix $\hat{M}_{0}$ in (3.4) is a non-singular M-matrix. Then, all solutions of (1.1) with initial conditions (1.2) are defined and bounded on $[0, \infty)$.

Proof. Solutions of (1.1) with initial conditions (1.2) are positive, whenever they are defined. For (1.1) written in the abstract form $X^{\prime}(t)=F\left(X_{t}\right)$, the function $F$ transforms bounded sets of $U C_{g}\left(\mathbb{R}^{2 n}\right)$ into bounded sets of $\mathbb{R}^{2 n}$, hence solutions are defined on compact intervals $[0, \alpha], \forall \alpha>0$, and therefore on $[0, \infty)$.

Since $\hat{M}_{0}$ is a non-singular M-matrix, there is a positive vector $\eta=\left(\eta_{1}, \ldots, \eta_{n}\right)$ such that $\hat{M}_{0} \eta>0[7]$, i.e.,

$$
\mu_{i} \eta_{i}>\sum_{j=1}^{n}\left|a_{i j}\right| \eta_{j}, \quad i=1, \ldots, n .
$$

Choose an arbitrarily small $\delta>0$ so that

$$
\mu_{i}-(1+\delta) \sum_{j=1}^{n}\left|a_{i j}\right| \frac{\eta_{j}}{\eta_{i}}>0, \quad i=1, \ldots, n,
$$

and a function $g$ for which (g1)-(g3) and (2.2) hold.

For $\bar{\eta}=\left(\eta_{1}^{-1}, \ldots, \eta_{n}^{-1}, e_{1}\left(d_{1} \eta_{1}\right)^{-1}, \ldots, e_{n}\left(d_{n} \eta_{n}\right)^{-1}\right)$, we further consider $\mathbb{R}^{2 n}$ equipped with the norm $|\cdot|_{\bar{\eta}}$ given by

$$
\left|\left(x_{1}, \ldots, x_{n}, u_{1}, \ldots, u_{n}\right)\right|_{\bar{\eta}}=\max \left\{\max _{i}\left(\frac{1}{\eta_{i}}\left|x_{i}\right|\right), \max _{i}\left(\frac{e_{i}}{\eta_{i} d_{i}}\left|u_{i}\right|\right)\right\} .
$$

Let $(x(t), u(t))=\left(x_{1}(t), \ldots, x_{n}(t), u_{1}(t), \ldots, u_{n}(t)\right)$ be a positive solution of (1.1). We claim that

$$
\sup _{t \geq 0}|(x(t), u(t))|_{\bar{\eta}}<\infty .
$$

For the sake of contradiction, assume that (3.7) fails. Then, for any $K>0$ there exists $T>0$ such that

$$
|(x(T), u(T))|_{\bar{\eta}} \geq|(K, \ldots, K)|_{\bar{\eta}} \quad \text { and } \quad|(x(T), u(T))|_{\bar{\eta}} \geq|(x(t), u(t))|_{\bar{\eta}}, 0 \leq t \leq T .
$$


Consider (3.8) with $K$ such that $|(K, \ldots, K)|_{\bar{\eta}}>\left\|\left(x_{0}, u_{0}\right)\right\|_{g, \bar{\eta}}=\sup _{s \leq 0} \frac{|(x(s), u(s))|_{\bar{\eta}}}{g(s)}$, and sufficiently large to be specified later.

If $|(x(T), u(T))|_{\bar{\eta}}=\frac{e_{i}}{\eta_{i} d_{i}} u_{i}(T)>\frac{1}{\eta_{i}} x_{i}(T)$ for some $i \in\{1, \ldots, n\}$, from (1.1) we obtain

$$
u_{i}^{\prime}(T)<-e_{i} u_{i}(T)+d_{i} \frac{e_{i}}{d_{i}} u_{i}(T) \leq 0
$$

which is not possible since the definition of $T$ implies $u_{i}^{\prime}(T) \geq 0$. Thus, $|(x(T), u(T))|_{\bar{\eta}}=\frac{1}{\eta_{i}} x_{i}(T)$ for some $i \in\{1, \ldots, n\}$. Clearly $x_{i}^{\prime}(T) \geq 0$.

Let $0 \leq t \leq T$ and $s \leq 0$. Note that $\frac{1}{\eta_{j}} \frac{x_{j}(t-s)}{g(-s)} \leq \frac{1}{\eta_{j}} \frac{x_{j}(t-s)}{g(t-s)}<\frac{1}{\eta_{j}} K \leq \frac{1}{\eta_{i}} x_{i}(T)$ if $t-s \leq 0$, and $\frac{1}{\eta_{j}} \frac{x_{j}(t-s)}{g(-s)} \leq \frac{1}{\eta_{i}} x_{i}(T)$ if $0 \leq t-s \leq T$. Hence

$$
\begin{aligned}
\eta_{j}^{-1}\left|a_{i j} \int_{0}^{\infty} K_{i j}(s) x_{j}(t-s) d s\right| & \leq \eta_{j}^{-1}\left|a_{i j}\right| \int_{0}^{\infty} g(-s) K_{i j}(s) \frac{x_{j}(t-s)}{g(-s)} d s \\
& <(1+\delta) \eta_{i}^{-1}\left|a_{i j}\right| x_{i}(T), j=1, \ldots, n .
\end{aligned}
$$

From (1.1) and (3.9), we obtain

$$
0 \leq x_{i}^{\prime}(T) \leq x_{i}(T)\left[b_{i}-\left(\mu_{i}-(1+\delta) \sum_{j=1}^{n}\left|a_{i j}\right| \frac{\eta_{j}}{\eta_{i}}\right) x_{i}(T)\right] .
$$

By (3.6), this is a contradiction if $K$ is chosen so that $K>b_{i}\left(\mu_{i}-(1+\delta) \sum_{j=1}^{n}\left|a_{i j}\right| \frac{\eta_{j}}{\eta_{i}}\right)^{-1}$.

In fact, a better criterion for the uniform boundedness of all positive solutions of (1.1) will be given later (cf. Theorem 3.3).

Note that $\hat{M}_{0}=\hat{M}+C$ where $C$ is a positive diagonal matrix. By Theorem 5.1.1 of [7], it follows that if $\hat{M}$ an M-matrix, then $\hat{M}_{0}$ is a non-singular M-matrix. Now, if $\hat{M}_{0}$ is a non-singular M-matrix, there is a positive vector $\eta=\left(\eta_{1}, \ldots, \eta_{n}\right)$ such that $\hat{M}_{0} \eta>0$, i.e., $\mu_{i} \eta_{i}>\sum_{j=1}^{n}\left|a_{i j}\right| \eta_{j}$ for $1 \leq i \leq n$ (cf. [7]); in particular, this implies 'diagonal dominance' of $M_{0}$, in the sense that $\left(\mu_{i}+a_{i i}\right) \eta_{i}>\sum_{j \neq i}\left|a_{i j}\right| \eta_{j}$ for $1 \leq i \leq n$. From [14, p. 201], it follows that if $\hat{M}_{0}$ is a non-singular M-matrix, then $-M_{0}$ (and hence $-M$ as well) is VL-stable, and therefore a P-matrix.

Lemma 3.2. Assume that the matrix $\hat{M}$ in (3.4) is an M-matrix, and that the unique saturated equilibrium $\left(x^{*}, u^{*}\right)$ of $(1.1)$ is positive. Then $\left(x^{*}, u^{*}\right)$ is locally asymptotically stable.

Proof. As observed, if $\hat{M}$ is an M-matrix then $M$ is a P-matrix, and there is a unique saturated equilibrium $\left(x^{*}, u^{*}\right)$. The linearisation of $(1.1)$ about $\left(x^{*}, u^{*}\right)$ is given by

$$
\left[\begin{array}{l}
y^{\prime}(t) \\
v^{\prime}(t)
\end{array}\right]=-\left(B\left[\begin{array}{l}
y(t) \\
v(t)
\end{array}\right]+\mathcal{L}\left[\begin{array}{l}
y_{t} \\
v_{t}
\end{array}\right]\right)
$$

with $y(t), v(t) \in \mathbb{R}^{n}$, and the $(2 n) \times(2 n)$ matrix $B$ and the linear operator $\mathcal{L}: B C_{g}\left(\mathbb{R}^{2 n}\right) \subset$ $U C_{g}\left(\mathbb{R}^{2 n}\right) \rightarrow \mathbb{R}^{2 n}$ defined below:

$$
B=\operatorname{diag}\left(\alpha_{1}, \ldots, \alpha_{n}, e_{1}, \ldots, e_{n}\right), \quad \mathcal{L}=\left(\mathcal{L}_{1}, \ldots, \mathcal{L}_{2 n}\right)
$$


where

$$
\alpha_{i}= \begin{cases}\mu_{i} x_{i}^{*}, & \text { if } x_{i}^{*}>0 \\ \sum_{j=1}^{n} a_{i j} x_{j}^{*}-b_{i}, & \text { if } x_{i}^{*}=0\end{cases}
$$

and

$$
\left\{\begin{array}{l}
\mathcal{L}_{i}(\varphi, \psi)=x_{i}^{*} \sum_{j=1}^{n} a_{i j} \int_{0}^{\infty} K_{i j}(s) \varphi_{j}(-s) d s+x_{i}^{*} c_{i} \int_{0}^{\infty} G_{i}(s) \psi_{i}(-s) d s, \\
\mathcal{L}_{n+i}(\varphi, \psi)=-d_{i} \varphi_{i}(0), \quad i=1, \ldots, n,
\end{array}\right.
$$

for $(\varphi, \psi)=\left(\varphi_{1}, \ldots, \varphi_{n}, \psi_{1}, \ldots, \psi_{n}\right)$. Note that $\alpha_{i} \geq 0$ for $1 \leq i \leq n$.

For $\left(\vec{e}_{1}, \ldots, \vec{e}_{2 n}\right)$ the canonical basis of $\mathbb{R}^{2 n}$, define $L:=B+\left[\mathcal{L}_{i}\left(\vec{e}_{j}\right)\right]_{i, j}$ and $\hat{L}:=B-$ $\left[\left|\mathcal{L}_{i}\left(\vec{e}_{j}\right)\right|\right]_{i, j}(1 \leq i, j \leq 2 n)$. We have

$$
\left[\mathcal{L}_{i}\left(\vec{e}_{j}\right)\right]_{i, j=1}^{n}=\left[\begin{array}{cc}
A\left(x^{*}\right) & C\left(x^{*}\right) \\
-D & 0
\end{array}\right]
$$

with $A\left(x^{*}\right)=\left[x_{i}^{*} a_{i j}\right]_{i, j}(1 \leq i, j \leq n), C\left(x^{*}\right)=\operatorname{diag}\left(x_{1}^{*} c_{1}, \ldots, x_{n}^{*} c_{n}\right)$ and $D=\operatorname{diag}\left(d_{1}, \ldots, d_{n}\right)$. Now suppose that $x^{*}>0$. It is easy to see that the matrices $L, \hat{L}$ are equivalent to, respectively,

$$
\left[\begin{array}{cc}
M\left(x^{*}\right) & C\left(x^{*}\right) \\
0 & E
\end{array}\right], \quad\left[\begin{array}{cc}
\hat{M}\left(x^{*}\right) & -C\left(x^{*}\right) \\
0 & E
\end{array}\right]
$$

where $E=\operatorname{diag}\left(e_{1}, \ldots, e_{n}\right), N\left(x^{*}\right)=\operatorname{diag}\left(\mu_{1} x_{1}^{*}, \ldots, \mu_{n} x_{n}^{*}\right), \tilde{C}\left(x^{*}\right)=\operatorname{diag}\left(x_{1}^{*} \frac{c_{1} d_{1}}{e_{1}}, \ldots, x_{n}^{*} \frac{c_{n} d_{n}}{e_{n}}\right)$, $M\left(x^{*}\right)=N\left(x^{*}\right)+A\left(x^{*}\right)+\tilde{C}\left(x^{*}\right)$ and $\hat{M}\left(x^{*}\right)=N\left(x^{*}\right)-A\left(x^{*}\right)-\tilde{C}\left(x^{*}\right)$. Note that $M\left(x^{*}\right), \hat{M}\left(x^{*}\right)$ are obtained from $M, \hat{M}$, respectively, by multiplying each line $i$ by $x_{i}^{*}$. Hence, it follows that $\operatorname{det} L \neq 0$ and that $\hat{L}$ is an M-matrix as well. From [4], we derive that the linear system (3.10) is exponentially asymptotically stable.

We remark that if $x_{i}^{*}=0$, then the $i$ th-line of the above matrix $\left[\mathcal{L}_{i}\left(\vec{e}_{j}\right)\right]$ is zero. Hence, a saturated equilibrium $\left(x^{*}, u^{*}\right)$ of $(1.1)$ on the boundary of the positive cone is not necessarily asymptotically stable, thus although its linearisation (3.10) is stable, one cannot deduce that $\left(x^{*}, u^{*}\right)$ is stable as a solution of (1.1).

Our main general result on the global attractivity of the saturated equilibrium is given below.

Theorem 3.2. Assume that the matrix $\hat{M}$ in (3.4) is an M-matrix. Then, there is a unique saturated equilibrium $\left(x^{*}, u^{*}\right)$ of (1.1), which is a global attractor of all solutions with initial conditions (1.2). Moreover, if $x^{*}>0$, then $\left(x^{*}, u^{*}\right)$ is GAS.

Proof. Since $\hat{M}$ is an M-matrix, from Theorem 3.1 and Lemma 3.1 we conclude that there is a unique saturated equilibrium $\left(x^{*}, u^{*}\right)$ of $(1.1)$ and that all positive solutions are defined and bounded on $[0, \infty)$. Lemma 3.2 shows that $\left(x^{*}, u^{*}\right)$ is stable if it is a positive equilibrium. We now need to show that $\left(x^{*}, u^{*}\right)$ is a global attractor of all positive solutions of (1.1).

Denote $I_{n}$ the $n \times n$ identity matrix. If $\hat{M}$ is an M-matrix, then for any $\delta_{0}>0$ the matrix $\delta_{0} I_{n}+\hat{M}$ is a non-singular M-matrix. Fix any $\delta_{0}>0$ and a positive vector $\eta=\left(\eta_{1}, \ldots, \eta_{n}\right)$ such that $\left(\delta_{0} I_{n}+\hat{M}\right) \eta>0$, i.e.,

$$
\left(\delta_{0}+\mu_{i}-c_{i} \frac{d_{i}}{e_{i}}\right) \eta_{i}>\sum_{j=1}^{n}\left|a_{i j}\right| \eta_{j}, \quad i=1, \ldots, n .
$$


Choose $\delta>0$ such that

$$
\left(\delta_{0}+\mu_{i}-c_{i} \frac{d_{i}}{e_{i}}\right) \eta_{i}-(1+\delta) \sum_{j=1}^{n}\left|a_{i j}\right| \eta_{j}>0, \quad 1 \leq i \leq n,
$$

and a function $g$ for which conditions (g1)-(g3) and (2.2) are fulfilled. We abuse the notation, and denote both norms in $\mathbb{R}^{2 n}$ and in $\mathbb{R}^{n}$ by $|\cdot|_{\bar{\eta}}$, where

$$
\begin{aligned}
\left|\left(x_{1}, \ldots, x_{n}, u_{1}, \ldots, u_{n}\right)\right|_{\bar{\eta}} & :=\max _{1 \leq i \leq n}\left\{\max \left(\frac{1}{\eta_{i}}\left|x_{i}\right|, \frac{e_{i}}{\eta_{i} d_{i}}\left|u_{i}\right|\right)\right\} \quad \text { in } \quad \mathbb{R}^{2 n}, \\
|x|_{\bar{\eta}} & :=\max _{1 \leq i \leq n} \frac{1}{\eta_{i}}\left|x_{i}\right| \quad \text { in } \quad \mathbb{R}^{n}
\end{aligned}
$$

and consider $U C_{g}\left(\mathbb{R}^{2 n}\right), U C_{g}\left(\mathbb{R}^{n}\right)$ equipped with the norms $\|\cdot\|_{g, \bar{\eta}}$.

Let $(x(t), u(t))$ be a positive solution of (1.1). With the change of variables

$$
y_{i}(t)=x_{i}(t)-x_{i}^{*}, v_{i}(t)=u_{i}(t)-u_{i}^{*}, \quad i=1, \ldots, n,
$$

system (1.1) together with definition 3.1 lead to

$$
\begin{aligned}
& y_{i}^{\prime}(t)=-\left(y_{i}(t)+x_{i}^{*}\right)\left(\mu_{i} y_{i}(t)+\sum_{j=1}^{n} a_{i j} \int_{0}^{\infty} K_{i j}(s) y_{j}(t-s) d s\right. \\
& \left.+c_{i} \int_{0}^{\infty} G_{i}(s) v_{i}(t-s) d s\right), \text { if } x_{i}^{*}>0, \\
& y_{i}^{\prime}(t) \leq-y_{i}(t)\left(\mu_{i} y_{i}(t)+\sum_{j=1}^{n} a_{i j} \int_{0}^{\infty} K_{i j}(s) y_{j}(t-s) d s\right. \\
& \left.+c_{i} \int_{0}^{\infty} G_{i}(s) v_{i}(t-s) d s\right), \text { if } x_{i}^{*}=0, \\
& v_{i}^{\prime}(t)=-e_{i} v_{i}(t)+d_{i} y_{i}(t), \quad i=1,2, \ldots, n .
\end{aligned}
$$

Define

$$
\liminf _{t \rightarrow \infty} y_{i}(t)=-l_{i}, \quad \limsup _{t \rightarrow \infty} y_{i}(t)=L_{i}, \quad i=1, \ldots, n
$$

and set

$$
l=\max _{1 \leq i \leq n} \frac{l_{i}}{\eta_{i}}, \quad L=\max _{1 \leq i \leq n} \frac{L_{i}}{\eta_{i}}, \quad U=\max (l, L) .
$$

Integrating (3.14), we get

$$
v_{i}(t)=v_{i}(0) e^{-e_{i} t}+d_{i} e^{-e_{i} t} \int_{0}^{t} e^{e_{i} s} y_{i}(s) d s, \quad t \geq 0,
$$

and therefore

$$
-x_{i}^{*} \leq-l_{i} \leq \frac{e_{i}}{d_{i}} \liminf _{t \rightarrow \infty} v_{i}(t) \leq \frac{e_{i}}{d_{i}} \limsup _{t \rightarrow \infty} v_{i}(t) \leq \mathrm{L}_{i}<\infty .
$$


Since $U \geq 0$, it is enough to prove that $U=0$. In order to get a contradiction, assume $U>0$.

Denote $I=\{1, \ldots, n\}, I_{1}=\left\{i \in I: \eta_{i}^{-1} L_{i}=U\right\}$ and $I_{2}=\left\{i \in I: \eta_{i}^{-1} l_{i}=U\right\}$. The assumption $U>0$ implies that $x_{i}^{*}>0$ if $i \in I_{2}$; otherwise, with $x_{i}^{*}=0$ we get $\liminf _{t \rightarrow \infty} x_{i}(t)=$ $\liminf _{t \rightarrow \infty} y_{i}(t)=-l_{i}=-\eta_{i} U \geq 0$, thus $U=0$.

The coordinates $y_{j}(t), v_{j}(t)$ are uniformly bounded for $t \geq 0$, thus, as remarked in Section 2, the positive orbit $\left\{\left(y_{t}, v_{t}\right): t \geq 0\right\}$ is precompact in $U C_{g}\left(\mathbb{R}^{2 n}\right)$.

Take any sequence $\left(t_{k}\right)$ with $t_{k} \rightarrow \infty$. Thus, there is a subsequence of $\left(y_{t_{k}}, v_{t_{k}}\right)$, still denoted by $\left(y_{t_{k}}, v_{t_{k}}\right)$, converging to some $(\phi, \psi)$ in $U C_{g}\left(\mathbb{R}^{2 n}\right)$. Let $\phi_{j}, \psi_{j}(1 \leq j \leq n)$ be the components of $\phi, \psi$, respectively. Take any $\varepsilon>0$ and let $t^{*}>0$ be such that $\eta_{j}^{-1}\left|y_{j}(t)\right| \leq U+\varepsilon$, for $t \geq$ $t^{*}, 1 \leq j \leq n$. For any $s \geq 0$, if $k$ is large so that $t_{k}-s \geq t^{*}$, then $\eta_{j}^{-1}\left|y_{t_{k}, j}(-s)\right| / g(-s) \mid=$ $\eta_{j}^{-1}\left|y_{j}\left(t_{k}-s\right)\right| / g(-s) \leq \eta_{j}^{-1}\left|y_{j}\left(t_{k}-s\right)\right| \leq U+\varepsilon$, therefore we get $\eta_{j}^{-1}\left\|\phi_{j}\right\|_{g} \leq U+\varepsilon$. In a similar way, we obtain $\eta_{j}^{-1} \frac{e_{i}}{d_{i}}\left\|\psi_{j}\right\|_{g} \leq U+\varepsilon$. Hence, we conclude that $\|(\phi, \psi)\|_{g, \bar{\eta}} \leq U$. Moreover, if $i \in I_{1} \cup I_{2}$ and $\left(t_{k}\right)$ is chosen in such a way that $\eta_{i}^{-1}\left|y_{i}\left(t_{k}\right)\right| \rightarrow U$, we further deduce that $\|\phi\|_{g, \bar{\eta}}=\eta_{i}^{-1}\left|\phi_{i}(0)\right|=U$ and that $y_{t_{k}, j}, v_{t_{k}, j}$ converge uniformly to $\phi_{j}, \psi_{j}$, respectively, on each compact set of $[0, \infty)$.

Fix $i \in I_{1} \cup I_{2}$. By the fluctuation lemma, take a sequence $\left(t_{k}\right)$ with $t_{k} \rightarrow \infty, y_{i}^{\prime}\left(t_{k}\right) \rightarrow 0$ and $\eta_{i}^{-1} y_{i}\left(t_{k}\right) \rightarrow\left\{\begin{array}{r}U, \text { if } i \in I_{1} \\ -U, \text { if } i \in I_{2}\end{array}\right.$. As above, we may assume that $\left(y_{t_{k}}, v_{t_{k}}\right) \rightarrow(\phi, \psi) \in U C_{g}\left(\mathbb{R}^{2 n}\right)$ for the norm $\|\cdot\|_{g, \bar{\eta}}$.

First, we consider the case $i \in I_{1}$, thus $\eta_{i}^{-1} y_{i}\left(t_{k}\right) \rightarrow U$.

Since the linear operator $\psi \mapsto \int_{0}^{\infty} G_{i}(s) \psi(-s) d s$, defined for $\psi \in B C(\mathbb{R}) \subset U C_{g}(\mathbb{R})$, is bounded, there exists

$$
\nu:=\lim _{k \rightarrow \infty} \int_{0}^{\infty} G_{i}(s) v_{i}\left(t_{k}-s\right) d s=\int_{0}^{\infty} G_{i}(s) \psi_{i}(-s) d s .
$$

From (3.17), we have $\left|\psi_{i}(-s)\right| \leq \eta_{i} \frac{d_{i}}{e_{i}} U$ for any $s \geq 0$, thus $\nu \geq-\eta_{i} \frac{d_{i}}{e_{i}} U$. From hypothesis (1.3), the inequality $\nu=-\eta_{i} \frac{d_{i}}{e_{i}} U$ implies that $\psi_{i}(0)=-\eta_{i} \frac{d_{i}}{e_{i}} U$. But, from (3.16) we have

$$
v_{i}\left(t_{k}\right)=v_{i}(0) e^{-e_{i} t_{k}}+d_{i} \int_{0}^{t_{k}} e^{-e_{i} u} y_{t_{k}, i}(-u) d u,
$$

and from the Lebesgue's dominated convergence theorem it follows that

$$
\lim _{k} v_{i}\left(t_{k}\right)=\psi_{i}(0)=d_{i} \int_{0}^{\infty} e^{-e_{i} u} \phi_{i}(-u) d u
$$

Since $\phi_{i}$ is a continuous function with $\eta_{i}^{-1}\left|\phi_{i}(-s)\right| \leq U$ for $s>0$ and $\eta_{i}^{-1} \phi_{i}(0)=U$, then $\eta_{i}^{-1} \int_{0}^{\infty} e^{-e_{i} s} \phi_{i}(-s) d s>-U / e_{i}$. We therefore conclude that

$$
\eta_{i}^{-1} \nu>-\frac{d_{i}}{e_{i}} U
$$

Moreover, in spite of the use of a specific vector $\eta=\eta\left(\delta_{0}\right)$ and norm $\|\cdot\|_{g, \bar{\eta}}$ in $U C_{g}\left(\mathbb{R}^{n}\right)$, obviously the limit $\nu$ does not depend on the chosen norm $|\cdot|_{\bar{\eta}}$ in $\mathbb{R}^{n}$. 
Next, denote

$$
H_{i}(t)=\mu_{i} y_{i}(t)+\sum_{j=1}^{n} a_{i j} \int_{0}^{\infty} K_{i j}(s) y_{j}(t-s) d s+c_{i} \int_{0}^{\infty} G_{i}(s) v_{i}(t-s) d s .
$$

From (3.12)-(3.13), we obtain

$$
y_{i}^{\prime}\left(t_{k}\right) \leq-\left(y_{i}\left(t_{k}\right)+x_{i}^{*}\right) H_{i}\left(t_{k}\right)
$$

From (2.2), we have (cf. (3.9))

$$
\left|a_{i j} \int_{0}^{\infty} K_{i j}(s) y_{j}\left(t_{k}-s\right) d s\right| \leq\left|a_{i j}\right| \int_{0}^{\infty} g(-s) K_{i j}(s) \frac{\left|y_{j}\left(t_{k}-s\right)\right|}{g(-s)} d s \leq(1+\delta)\left|a_{i j}\right|\left\|y_{t_{k}, j}\right\|_{g}
$$

and this leads to

$$
\begin{aligned}
H_{i}\left(t_{k}\right) & \geq \mu_{i} y_{i}\left(t_{k}\right)-(1+\delta) \sum_{j=1}^{n}\left|a_{i j}\right|\left\|y_{t_{k}, j}\right\|_{g, \bar{\eta}}+c_{i} \int_{0}^{\infty} G_{i}(s) v_{i}\left(t_{k}-s\right) d s \\
& \geq \mu_{i} y_{i}\left(t_{k}\right)-(1+\delta) \sum_{j=1}^{n}\left|a_{i j}\right| \eta_{j}\left\|y_{t_{k}}\right\|_{g, \bar{\eta}}+c_{i} \int_{0}^{\infty} G_{i}(s) v_{i}\left(t_{k}-s\right) d s .
\end{aligned}
$$

By letting $k \rightarrow \infty$, from (3.11) and (3.21) we have

$$
0 \geq\left(\mu_{i} \eta_{i}-(1+\delta) \sum_{j=1}^{n}\left|a_{i j}\right| \eta_{j}\right) U+c_{i} \nu \geq\left(c_{i} \frac{d_{i}}{e_{i}}-\delta_{0}\right) \eta_{i} U+c_{i} \nu
$$

Since $\delta_{0}>0$ is arbitrarily small, this yields $\nu \leq-\frac{d_{i}}{e_{i}} \eta_{i} U$, which is not possible in view of (3.18).

Now, consider the case $i \in I_{2}$. Then, $\eta_{i}^{-1} y_{i}\left(t_{k}\right) \rightarrow-U$ and (3.12) holds.

If $y_{i}(t)$ is eventually monotone, then $y_{i}(t) \rightarrow-\eta_{i} U$ and $v_{i}(t) \rightarrow-\frac{d_{i}}{e_{i}} \eta_{i} U$. Using arguments similar to the ones above, we obtain

$$
\begin{aligned}
H_{i}\left(t_{k}\right) \leq \mu_{i} y_{i}\left(t_{k}\right) & +(1+\delta) \sum_{j=1}^{n}\left|a_{i j}\right| \eta_{j}\left\|y_{t_{k}}\right\|_{g, \bar{\eta}}+c_{i} \int_{0}^{\infty} G_{i}(s) v_{i}\left(t_{k}-s\right) d s \\
& \rightarrow\left[-\left(\mu_{i}+c_{i} \frac{d_{i}}{e_{i}}\right) \eta_{i}+(1+\delta) \sum_{j=1}^{n}\left|a_{i j}\right| \eta_{j}\right] U<0 .
\end{aligned}
$$

Since $y_{i}^{\prime}\left(t_{k}\right)=-\left(y_{i}\left(t_{k}\right)+x_{i}^{*}\right) H_{i}\left(t_{k}\right)$, using the above estimate we obtain

$$
0 \geq\left(-\eta_{i} U+x_{i}^{*}\right)\left[\left(\mu_{i}+c_{i} \frac{d_{i}}{e_{i}}\right) \eta_{i}-(1+\delta) \sum_{j=1}^{n}\left|a_{i j}\right| \eta_{j}\right] U
$$

and thus $y_{i}(t) \rightarrow-x_{i}^{*}=-\eta_{i} U$ as $t \rightarrow \infty$. Since $y_{i}(t)>-x_{i}^{*}$ for $t>0$, this is only possible if $y_{i}^{\prime}(t) \leq 0$ for $t$ large, so that $\eta_{i}^{-1} y_{i}(t) \searrow-U$. But in this case from (3.12) it follows that $H_{i}(t) \geq 0$ for $t$ large, which contradicts (3.23). 
If $y_{i}(t)$ is not eventually monotone, then we can assume that $y_{i}\left(t_{k}\right)$ is a sequence of minima, so that $H_{i}\left(t_{k}\right)=0$, and this case is treated as the case $i \in I_{1}$. These arguments show that $U=0$, and the proof is complete.

Remark 3.3. As referred to in the introduction, clearly the above proof applies to systems (1.4). In fact, with the terms $a_{i j} \int_{0}^{\infty} K_{i j}(s) x_{j}(t-s) d s, c_{i} \int_{0}^{\infty} G_{i}(s) u_{i}(t-s) d s$ replaced by the more general linearities

$$
a_{i j} \int_{0}^{\infty} x_{j}(t-s) d \eta_{i j}(s), \quad c_{i} \int_{0}^{\infty} u_{i}(t-s) d \nu_{i}(s),
$$

where $\eta_{i j}, \nu_{i}$ are normalized bounded variation functions and $\nu_{i}$ are non-decreasing, we use $(2.3)$ instead of (2.2), the estimates (3.20) are replaced by

$$
\begin{aligned}
\left|a_{i j} \int_{0}^{\infty} y_{j}\left(t_{k}-s\right) d \eta_{i j}(s)\right| & \leq\left|a_{i j}\right| \int_{0}^{\infty} g(-s) \frac{\left|y_{j}\left(t_{k}-s\right)\right|}{g(-s)} d\left|\eta_{i j}(s)\right| \\
& \leq(1+\delta)\left|a_{i j}\right|\left\|y_{t_{k}, j}\right\|_{g}, \quad i, j=1, \ldots, n,
\end{aligned}
$$

the limit $\nu$ is now given by $\nu=\int_{0}^{\infty} \psi_{i}(-s) d \nu_{i}(s)$, and all the other arguments are valid.

With the usual notation of

$$
a_{i j}=a_{i j}^{+}-a_{i j}^{-}, \quad \text { where } a_{i j}^{+}=\max \left\{a_{i j}, 0\right\}, a_{i j}^{-}=\max \left\{-a_{i j}, 0\right\},
$$

we denote

$$
M_{0}^{-}=\operatorname{diag}\left(\mu_{1}, \ldots, \mu_{n}\right)-A^{-}, \quad \text { where } A^{-}=\left[a_{i j}^{-}\right] .
$$

Note that $M_{0}^{-} \geq \hat{M}_{0}$, hence in general imposing that $M_{0}^{-}$is a non-singular M-matrix is weaker than requiring that $\hat{M}_{0}$ is a non-singular M-matrix. We now give sufficient conditions for the dissipativeness of (1.1), improving Lemma 3.1.

Theorem 3.3. If $M_{0}^{-}$is a non-singular M-matrix, then (1.1) is dissipative; i.e., there exists $K>0$ such that $\lim \sup _{t \rightarrow \infty} x_{i}(t) \leq K, \lim \sup _{t \rightarrow \infty} u_{i}(t) \leq K, 1 \leq i \leq n$, for all solutions $(x(t), u(t))$ of (1.1) with initial conditions (1.2).

Proof. A solution $(x(t), u(t))$ of $(1.1)$ with initial condition $\left(x_{0}, u_{0}\right)=(\varphi, \psi) \in B C_{0}^{+}$satisfies

$$
\left\{\begin{array}{l}
x_{i}^{\prime}(t) \leq x_{i}(t)\left(b_{i}-\mu_{i} x_{i}(t)+\sum_{j=1}^{n} a_{i j}^{-} \int_{0}^{\infty} K_{i j}(s) x_{j}(t-s) d s\right) \\
u_{i}^{\prime}(t)=-e_{i} u_{i}(t)+d_{i} x_{i}(t), \quad i=1,2, \ldots, n
\end{array}\right.
$$

Let $(X(t), U(t))$ be the solution of the system

$$
\left\{\begin{array}{l}
X_{i}^{\prime}(t)=X_{i}(t)\left(b_{i}-\mu_{i} X_{i}(t)+\sum_{j=1}^{n} a_{i j}^{-} \int_{0}^{\infty} K_{i j}(s) X_{j}(t-s) d s\right) \\
U_{i}^{\prime}(t)=-e_{i} U_{i}(t)+d_{i} X_{i}(t), \quad i=1,2, \ldots, n
\end{array}\right.
$$

with the initial conditions $X_{0}=\varphi, U(0)=\psi(0)$. Since $(3.25)$ is cooperative, or in other worths, it satisfies the quasi-monotonicity condition in [22, Chapter 5], by comparison results it follows 
that $x(t) \leq X(t), u(t) \leq U(t)$. From [4, Corollary 4.1], $(X(t), U(t)) \rightarrow\left(X^{*}, U^{*}\right)$ as $t \rightarrow \infty$, where $\left(X^{*}, U^{*}\right)$ is the saturated equilibrium of (3.25). Thus, the solutions $(x(t), u(t))$ of the initial value problems (1.1)-(1.2) satisfy $\lim \sup _{t \rightarrow \infty} x_{i}(t) \leq X_{i}^{*}, \limsup _{t \rightarrow \infty} u_{i}(t) \leq U_{i}^{*}, 1 \leq i \leq n$.

Our setting contemplates all the possibilities for the signs of the coefficients $b_{i}, a_{i j}$ in (3.1). In biological terms, the most interesting cases are however: (i) $a_{i j} \geq 0$ for $i \neq j$ (competitive systems); (ii) $a_{i j} \leq 0$ for $i \neq j$ (cooperative systems); (iii) $a_{i j}>0, a_{j i}<0$ (predator-prey systems) if species $i$ is a prey for the predator species $j, i \neq j$. On the other hand, the existence of a positive equilibrium depends heavily on the coefficients $b_{i}$ 's, and can be studied in more detail by using Cramer's rule. Nevertheless, a criterion for cooperative systems is given here.

Theorem 3.4. Consider (1.1) with $b_{i}>0$ and $a_{i j} \leq 0$ for all $i \neq j$. If $M$ is a non-singular M-matrix, then there exists a unique positive equilibrium of (1.1).

Proof. Denote $b=\left(b_{1}, \ldots, b_{n}\right)$. Since $M$ is non-singular M-matrix, then $M^{-1} \geq 0$ [1]. This implies that $\left(M^{-1} b\right)_{i}=0$ if and only if the $i$ th line of $M^{-1}$ is zero, which is not possible. Therefore, $x^{*}:=M^{-1} b$ is a positive vector, and $\left(x^{*}, u^{*}\right)$, with $u_{i}^{*}=\frac{d_{i}}{e_{i}} x_{i}^{*}, 1 \leq i \leq n$, is a positive equilibrium of (1.1).

\section{Extinction and stability}

For the results in this section, it is important to consider systems (1.1) with $K_{i j}$ non-negative, or more general systems (1.4) with $\eta_{i j}$ non-decreasing, $i, j=1, \ldots, n$. Straightforward generalisations for the situation of $K_{i j}$ in (1.1) changing signs or $\eta_{i j}$ non-monotone on $[0, \infty)$ can however be derived (cf. [4] for the case of uncontrolled Lotka-Volterra models).

We now seek for better sufficient conditions for extinction of either all or part of the populations. Together with the controlled Lotka-Volterra system (1.1), consider the ODE system (3.5), and write (3.5) in the form $X^{\prime}(t)=F(X(t))$ for $X(t)=\left(x_{1}(t), \ldots, x_{n}(t), u_{1}(t), \ldots, u_{n}(t)\right)$.

Define $\lambda_{i}=\mu_{i}+\frac{c_{i} d_{i}}{e_{i}}$. If $X^{*}=\left(x^{*}, u^{*}\right)=\left(x_{1}^{*}, \ldots, x_{n}^{*}, u_{1}^{*}, \ldots, u_{n}^{*}\right)$ is an equilibrium of (3.5), then

$$
D F\left(X^{*}\right)=\left[\begin{array}{cc}
D f\left(x^{*}\right) & -C\left(x^{*}\right) \\
D & -E
\end{array}\right]
$$

where $C\left(x^{*}\right)=\operatorname{diag}\left(c_{1} x_{1}^{*}, \ldots, c_{n} x_{n}^{*}\right), D=\operatorname{diag}\left(d_{1}, \ldots, d_{n}\right), E=\operatorname{diag}\left(e_{1}, \ldots, e_{n}\right)$, and $\frac{\partial f_{i}}{\partial x_{i}}\left(x^{*}\right)=$ $b_{i}-\left(\lambda_{i}+a_{i i}\right) x_{i}^{*}-\sum_{j=1}^{n} a_{i j} x_{j}^{*}-\left(\mu_{i}+a_{i i}\right) x_{i}^{*}, \frac{\partial f_{i}}{\partial x_{j}}\left(x^{*}\right)=-a_{i j} x_{i}^{*}$ if $i \neq j$. Note that $\frac{\partial f_{i}}{\partial x_{i}}\left(x^{*}\right)=$ $-\left(\mu_{i}+a_{i i}\right) x_{i}^{*}$ if $x_{i}^{*}>0$, otherwise $\frac{\partial f_{i}}{\partial x_{i}}\left(x^{*}\right) \leq-\left(\mu_{i}+a_{i i}\right) x_{i}^{*}$.

For the trivial equilibrium, we have $C(0)=0$, hence the spectrum of $D F(0)$ is $\sigma(D F(0))=$ $\left\{b_{1}, \ldots, b_{n},-e_{1}, \ldots,-e_{n}\right\}$. We therefore conclude that zero is a stable equilibrium for the linearisation of (3.5) at zero (which is also the linearisation of (1.1)) if and only if $b_{i} \leq 0,1 \leq i \leq n$, and the introduction of the controls does not change its stability.

Let $M$ be a P-matrix. If $b_{i} \leq 0,1 \leq i \leq n$, then 0 is the saturated equilibrium; on reverse, if $b_{i}>0$ for some $i$, then $D F(0)$ is unstable and zero is not the saturated equilibrium. In the latter case, we have seen that (1.1) is dissipative if $M_{0}^{-}$is a non-singular M-matrix - then there 
exists a compact global attractor ([11, Theorem 3.4.8]), which however need not be the saturated equilibrium. When 0 is saturated, rather than Theorem 3.2, next result provides a better criterion for extinction of all populations.

Theorem 4.1. Assume that $M$ is a $P$-matrix. The equilibrium 0 is the saturated equilibrium of (1.1) if and only if $b_{i} \leq 0$ for $1 \leq i \leq n$. In this case, if $M_{0}^{-}$is an $M$-matrix, where $M_{0}^{-}$is defined as in (3.24), then the equilibrium 0 of (1.1) is globally attractive.

Proof. Since $M_{0}^{-}$is an M-matrix, for any arbitrarily small $\delta_{0}>0$, consider a positive vector $\eta=\left(\eta_{1}, \ldots, \eta_{n}\right)$ such that $\left(M_{0}^{-}+\delta_{0} I_{n}\right) \eta>0[7]$. Let $(x(t), u(t))$ be a solution of (1.1). After a scaling $x_{i} \mapsto \bar{x}_{i}=\eta_{i}^{-1} x_{i}, u_{i} \mapsto \bar{u}_{i}=\eta_{i}^{-1} u_{i}, 1 \leq i \leq n$, and dropping the bars for the sake of simplicity, we may suppose that $(x(t), u(t))$ is a solution of (1.1) and that $\left(M_{0}^{-}+\delta_{0} I_{n}\right) \eta>0$ with $\eta=(1, \ldots, 1)$. Next, choose $\delta>0$ small and $g$ satisfying (g1)-(g3) and (2.2), with

$$
\delta_{0}+\mu_{i}-(1+\delta) \sum_{j=1}^{n} a_{i j}^{-}>0, \quad 1 \leq i \leq n .
$$

Define $L_{i}=\limsup _{t \rightarrow \infty} x_{i}(t)$ and $U=\max _{1 \leq i \leq n} L_{i}$. For the sake of contradiction, assume that $U>0$, and choose $i \in\{1, \ldots, n\}$ such that $L_{i}=U$. Consider a sequence $\left(t_{k}\right)$ with $t_{k} \rightarrow \infty$, $x_{i}^{\prime}\left(t_{k}\right) \rightarrow 0, x_{i}\left(t_{k}\right) \rightarrow U$ as $k \rightarrow \infty$. We now argue as in the proof of Theorem 3.2, omitting some of the details. For some subsequence of $\left(x_{t_{k}}, u_{t_{k}}\right)$, still denoted by $\left(x_{t_{k}}, u_{t_{k}}\right)$, there is $(\phi, \psi) \in$ $B C^{+}\left(\mathbb{R}^{2 n}\right) \subset U C_{g}\left(\mathbb{R}^{2 n}\right)$ such that $x_{t_{k}} \rightarrow \phi, u_{t_{k}} \rightarrow \psi$, and $\|\phi\|_{g}=U=\phi_{i}(0)$. Next, from (1.3) and the fact that $\psi_{i}(0)=d_{i} \int_{0}^{\infty} e^{-e_{i} u} \phi_{i}(-u) d u>0$, we obtain $\nu:=\lim _{k \rightarrow \infty} \int_{0}^{\infty} G_{i}(s) u_{i}\left(t_{k}-s\right) d s=$ $\int_{0}^{\infty} G_{i}(s) \psi_{i}(-s) d s>0$. Choose $\delta_{0}>0$ small and $k$ large so that $c_{i} \int_{0}^{\infty} G_{i}(s) u_{i}\left(t_{k}-s\right) d s>\delta_{0} U$. Since $b_{i} \leq 0$, for $k$ large estimates as in (3.9) yield

$$
\begin{aligned}
x_{i}^{\prime}\left(t_{k}\right) & \leq x_{i}\left(t_{k}\right)\left(b_{i}-\mu_{i} x_{i}\left(t_{k}\right)+\sum_{j=1}^{n} a_{i j}^{-} \int_{0}^{\infty} K_{i j}(s) x_{j}\left(t_{k}-s\right) d s-c_{i} \int_{0}^{\infty} G_{i}(s) u_{i}\left(t_{k}-s\right) d s\right) \\
& \leq-x_{i}\left(t_{k}\right)\left(\mu_{i} x_{i}\left(t_{k}\right)-\sum_{j=1}^{n} a_{i j}^{-}\left\|x_{t_{k}, j}\right\|_{g} \int_{0}^{\infty} g(-s) K_{i j}(s) d s+\delta_{0} U\right) \\
& \leq-x_{i}\left(t_{k}\right)\left(\mu_{i} x_{i}\left(t_{k}\right)-(1+\delta) \sum_{j=1}^{n} a_{i j}^{-}\left\|x_{t_{k}, j}\right\|_{g}+\delta_{0} U\right) .
\end{aligned}
$$

By letting $k \rightarrow \infty$ we obtain

$$
0 \geq\left[\delta_{0}+\mu_{i}-(1+\delta) \sum_{j=1}^{n} a_{i j}^{-}\right] U
$$

which contradicts (4.1). Hence $U=0$, and the proof is complete.

Consider now the case of a saturated equilibrium $\left(x^{*}, u^{*}\right) \neq 0$ of $(1.1)$ with $x^{*} \in \partial\left(\mathbb{R}_{+}^{n}\right)$. By reordering the variables, write $x^{*}=\left(x_{1}^{*}, \ldots, x_{p}^{*}, 0, \ldots, 0\right)$ with $x_{i}^{*}>0$ for $1 \leq i \leq p$, where $1<p<$ $n$. Here, the attractivity of $\left(x^{*}, u^{*}\right)$ means the extinction of the populations $x_{i}(t), p+1 \leq j \leq n$, while the first $p$ populations $x_{i}(t)$ stabilize with time at the 'saturated' value $x_{i}^{*}$. For this situation, next result improves Theorem 3.2. Its statement includes Theorems 3.2 and 4.1 as particular cases. 
Theorem 4.2. Assume that $M$ is a P-matrix, let $\left(x^{*}, u^{*}\right)$ be the saturated equilibrium of (1.1), and suppose that $x^{*}=\left(x_{1}^{*}, \ldots, x_{p}^{*}, 0, \ldots, 0\right)(0 \leq p \leq n)$. Write $n_{1}=p, n_{2}=n-p$, and the matrices $A=\left[a_{i j}\right],|A|=\left[\left|a_{i j}\right|\right], A^{-}=\left[a_{i j}^{-}\right]$in the form

$$
A=\left[\begin{array}{ll}
A_{11} & A_{12} \\
A_{21} & A_{22}
\end{array}\right], \quad|A|=\left[\begin{array}{ll}
\left|A_{11}\right| & \left|A_{12}\right| \\
\left|A_{21}\right| & \left|A_{22}\right|
\end{array}\right], \quad A^{-}=\left[\begin{array}{cc}
A_{11}^{-} & A_{12}^{-} \\
A_{21}^{-} & A_{22}^{-}
\end{array}\right]
$$

where $A_{k l},\left|A_{k l}\right|, A_{k l}^{-}$are $n_{k} \times n_{l}$ matrices for $k, l=1,2$. Define also

$$
\hat{\mathcal{M}}_{11}=\operatorname{diag}\left(\mu_{1}-c_{1} \frac{d_{1}}{e_{1}}, \ldots, \mu_{p}-c_{p} \frac{d_{p}}{e_{p}}\right)-\left|A_{11}\right|, \quad \mathcal{M}_{22}^{-}=\operatorname{diag}\left(\mu_{p+1}, \ldots, \mu_{n}\right)-A_{22}^{-} .
$$

If the matrix

$$
\hat{\mathcal{M}}:=\left[\begin{array}{cc}
\hat{\mathcal{M}}_{11} & -\left|A_{12}\right| \\
-\left|A_{21}\right| & \mathcal{M}_{22}^{-}
\end{array}\right]
$$

is an M-matrix, then $\left(x^{*}, u^{*}\right)$ is a global attractor for the solutions $(x(t), u(t))$ of $(1.1)-(1.2)$.

Proof. The cases $p=n$ and $p=0$ were treated in Theorems 3.2 and 4.1, respectively. Now, consider $0<p<n$. Again, the proof follows along the lines of the proof of Theorem 3.2, so some details are omitted.

Assume that $\hat{\mathcal{M}}$ is an M-matrix. Choose an arbitrarily small $\delta_{0}>0$. Since $\delta_{0} I_{n}+\hat{\mathcal{M}}$ is a non-singular M-matrix, there is a positive vector $\eta$ such that $\left(\delta_{0} I_{n}+\hat{\mathcal{M}}\right) \eta>0$. After a scaling $x_{i} \mapsto \bar{x}_{i}=\eta_{i}^{-1} x_{i}, u_{i} \mapsto \bar{u}_{i}=\eta_{i}^{-1} u_{i}, 1 \leq i \leq n$, and dropping the bars for the sake of simplicity, we may suppose that $\eta=(1, \ldots, 1)$. Choose $\delta>0$ small and $g$ satisfying (g1)-(g3) and (2.2), with

$$
\begin{gathered}
\delta_{0}+\mu_{i}-c_{i} \frac{d_{i}}{e_{i}}>(1+\delta) \sum_{j=1}^{n}\left|a_{i j}\right|, \quad 1 \leq i \leq n_{1}, \\
\delta_{0}+\mu_{i}>(1+\delta)\left(\sum_{j=1}^{n_{1}}\left|a_{i j}\right|+\sum_{j=n_{1}+1}^{n} a_{i j}^{-}\right), \quad n_{1}+1 \leq i \leq n .
\end{gathered}
$$

For $i>n_{1}$, let $\alpha_{i} \geq 0$ be such that $b_{i}+\alpha_{i}=\sum_{j=1}^{n_{1}} a_{i j} x_{j}^{*}$, and define $\alpha_{i}=0$ for $1 \leq i \leq n_{1}$. We now effect the changes $y_{i}(t)=x_{i}(t)-x_{i}^{*}, v_{i}(t)=u_{i}(t)-u_{i}^{*}$ for $1 \leq i \leq n$, so we keep $y_{i}(t)=x_{i}(t), v_{i}(t)=u_{i}(t)$ for $n_{1}+1 \leq i \leq n$. Besides (3.14), we obtain

$$
y_{i}^{\prime}(t)=-\left(y_{i}(t)+x_{i}^{*}\right) H_{i}(t), \quad 1 \leq i \leq n,
$$

where now

$$
H_{i}(t)=\alpha_{i}+\mu_{i} y_{i}(t)+\sum_{j=1}^{n} a_{i j} \int_{0}^{\infty} K_{i j}(s) y_{j}(t-s) d s+c_{i} \int_{0}^{\infty} G_{i}(s) v_{i}(t-s) d s, \quad 1 \leq i \leq n
$$

Define $l_{i}, L_{i}$ as in (3.15), and recall that $0 \leq-l_{i} \leq L_{i}$ for $i>n_{1}$. Set $l=\max _{1 \leq i \leq n_{1}} l_{i}, L=$ $\max _{1 \leq i \leq n} L_{i}$. We need to prove that $U:=\max (l, L)=0$. 
For any $\varepsilon>0$ small, if $t>0$ is sufficiently large we have

$$
\begin{gathered}
\left|a_{i j} \int_{0}^{\infty} K_{i j}(s) y_{j}(t-s) d s\right| \leq\left|a_{i j}\right|\left(\max \left(l_{j}, L_{j}\right)+\varepsilon\right), \quad 1 \leq i, j \leq n, \\
\int_{0}^{\infty} K_{i j}(s) y_{j}(t-s) d s \geq-a_{i j}^{-}\left(L_{j}+\varepsilon\right), \quad n_{1}+1 \leq j \leq n .
\end{gathered}
$$

Suppose that $U>0$. If $U=L_{i}$ or $U=l_{i}$ for some $i \in\left\{1, \ldots, n_{1}\right\}$, we choose a sequence $t_{k} \rightarrow \infty$ with $y_{i}^{\prime}\left(t_{k}\right) \rightarrow 0, y_{i}\left(t_{k}\right) \rightarrow L_{i}$, respectively $y_{i}\left(t_{k}\right) \rightarrow-l_{i}$, and $\left(y_{t_{k}}, v_{t_{k}}\right) \rightarrow(\phi, \psi) \in B C \subset$ $U C_{g}$ as $k \rightarrow \infty$. If $U=l_{i}>0$ for some $i \in\left\{1, \ldots, n_{1}\right\}$ and $y_{i}(t)$ is eventually monotone, we proceed as in the proof of Theorem 3.2 and easily get a contradiction. Otherwise, $\left(t_{k}\right)$ may be chosen so that $H_{i}\left(t_{k}\right)=0$. We argue as in the proof of Theorem 3.2, and obtain the estimates (3.21), (3.23), respectively (where now we suppose that $\eta_{j}=1$ for all $j$ ). As in (3.18), we obtain $\nu:=\lim _{k \rightarrow \infty} \int_{0}^{\infty} G_{i}(s) v_{i}\left(t_{k}-s\right) d s>-d_{i} L_{i} / e_{i}$ if $y_{i}\left(t_{k}\right) \rightarrow L_{i}$, and $\nu<d_{i} l_{i} / e_{i}$ if $y_{i}\left(t_{k}\right) \rightarrow-l_{i}$, so we may suppose that $\delta_{0}>0$ in (4.3)-(4.4) was chosen so that $c_{i} \nu / L_{i}>-c_{i} d_{i} / e_{i}+\delta_{0}$, respectively $c_{i} \nu / l_{i}<c_{i} d_{i} / e_{i}-\delta_{0}$. By taking limits $k \rightarrow \infty, \varepsilon_{0} \rightarrow 0^{+}$, we derive

$$
0 \geq\left(\mu_{i}-(1+\delta) \sum_{j=1}^{n}\left|a_{i j}\right|\right) U+c_{i} \nu
$$

a contradiction with (4.3).

If $U=L_{i}$ for some $i \in\left\{n_{1}+1, \ldots, n\right\}$, we choose a sequence $t_{k} \rightarrow \infty$ with $y_{i}\left(t_{k}\right) \rightarrow L_{i}, y_{i}^{\prime}\left(t_{k}\right) \rightarrow$ 0 , proceed as above, and obtain

$$
0 \geq\left(\alpha_{i}+\mu_{i}-(1+\delta) \sum_{j=1}^{n_{1}}\left|a_{i j}\right|-(1+\delta) \sum_{j=n_{1}+1}^{n} a_{i j}^{-}\right) U+c_{i} \nu,
$$

where now $0 \leq \phi_{i}(-s) \leq U, \phi_{i}(0)=U>0$, thus $d_{i} \int_{0}^{\infty} e^{-e_{i} s} \phi_{i}(-s) d s>0$, which implies that $\nu:=\lim _{k \rightarrow \infty} \int_{0}^{\infty} G_{i}(s) v_{i}\left(t_{k}-s\right) d s>0$. Therefore, the above estimate contradicts (4.4).

For equilibria on the boundary of $\mathbb{R}_{+}^{n}$, and depending on the sizes and signs of coefficients $b_{i}^{\prime} \mathrm{s}$, one might be able to slightly improve the conditions in Theorem 4.2.

Theorem 4.3. Assume that $M$ is a P-matrix, let $\left(x^{*}, u^{*}\right)$ be the saturated equilibrium of (1.1), and suppose that $x^{*}=\left(x_{1}^{*}, \ldots, x_{p}^{*}, 0, \ldots, 0\right)(1 \leq p<n)$. Besides the notations in the statement of Theorem 4.2, we further denote $\mathcal{A}_{21}=\left[\tilde{a}_{i j}\right]$, where

$$
\tilde{a}_{i j}=\left\{\begin{array}{ll}
a_{i j}^{-}, & \text {if } b_{i}+\sum_{j=1}^{p} a_{i j}^{-} x_{j}^{*} \leq 0 \\
\left|a_{i j}\right|, & \text { if } b_{i}+\sum_{j=1}^{p} a_{i j}^{-} x_{j}^{*}>0
\end{array}, \quad i=p+1, \ldots, n, j=1, \ldots, p .\right.
$$

If

$$
\hat{\mathcal{M}}:=\left[\begin{array}{cc}
\hat{\mathcal{M}}_{11} & -\left|A_{12}\right| \\
-\mathcal{A}_{21} & \mathcal{M}_{22}^{-}
\end{array}\right]
$$

is an M-matrix, then $\left(x^{*}, u^{*}\right)$ is a global attractor for the solutions $(x(t), u(t))$ of (1.1)-(1.2). 
In particular, if $A_{21} \geq 0, b_{i} \leq 0$ for $p<i \leq n$, and $\hat{\mathcal{M}}_{11}$ and $\mathcal{M}_{22}^{-}$are M-matrices, then $\left(x^{*}, u^{*}\right)$ is globally attractive.

Proof. Set $p=n_{1}, n-p=n_{2}, y_{i}(t)=x_{i}(t)-x_{i}^{*}, v_{i}(t)=u_{i}(t)-u_{i}^{*}$ for $1 \leq i \leq n$. For each $i>n_{1}$, the function $H_{i}(t)$ in (4.5) is given by

$$
\begin{aligned}
& H_{i}(t)=\alpha_{i}+\mu_{i} y_{i}(t)+\sum_{j=1}^{n_{1}} a_{i j} \int_{0}^{\infty} K_{i j}(s) y_{j}(t-s) d s \\
& +\sum_{j=n_{1}+1}^{n} a_{i j} \int_{0}^{\infty} K_{i j}(s) y_{j}(t-s) d s+c_{i} \int_{0}^{\infty} G_{i}(s) v_{i}(t-s) d s \\
& \geq \alpha_{i}-\sum_{i=1}^{n_{1}} a_{i j}^{+} x_{j}^{*}+\mu_{i} y_{i}(t)-\sum_{j=1}^{n} a_{i j}^{-} \int_{0}^{\infty} K_{i j}(s) y_{j}(t-s) d s+c_{i} \int_{0}^{\infty} G_{i}(s) v_{i}(t-s) d s \\
& =-\left(b_{i}+\sum_{j=1}^{n_{1}} a_{i j}^{-} x_{j}^{*}\right)+\mu_{i} y_{i}(t)-\sum_{j=1}^{n} a_{i j}^{-} \int_{0}^{\infty} K_{i j}(s) y_{j}(t-s) d s+c_{i} \int_{0}^{\infty} G_{i}(s) v_{i}(t-s) d s .
\end{aligned}
$$

For each $i>n_{1}$, we can use the arguments in the above proof, with formula (4.6) replaced by the above estimate if $\left(b_{i}+\sum_{j=1}^{n_{1}} a_{i j}^{-} x_{j}^{*}\right) \leq 0$.

Now, if $A_{21} \geq 0$ and $b_{i} \leq 0$ for $p<i \leq n$, the matrix in (4.8) becomes $\hat{\mathcal{M}}=\left[\begin{array}{cc}\hat{\mathcal{M}}_{11} & -\left|A_{12}\right| \\ 0 & \mathcal{M}_{22}^{-}\end{array}\right]$, which is an M-matrix if and only if $\hat{\mathcal{M}}_{11}$ and $\mathcal{M}_{22}^{-}$are M-matrices.

In applications, the following corollary is also useful.

Corollary 4.1. Assume that $M$ is a P-matrix, let $\left(x^{*}, u^{*}\right)$ be the unique saturated equilibrium of (1.1), and $h_{i}:[0, \infty) \rightarrow \mathbb{R}$ continuous functions with $h_{i}(t) \rightarrow 0$ as $t \rightarrow \infty(1 \leq i \leq n)$. Under the assumptions of Theorems 3.2, 4.2 or 4.3, then all solutions $(x(t), u(t))$ of

$$
\left\{\begin{array}{l}
x_{i}^{\prime}(t)=x_{i}(t)\left(b_{i}-\mu_{i} x_{i}(t)-\sum_{j=1}^{n} a_{i j} \int_{0}^{\infty} K_{i j}(s) x_{j}(t-s) d s-c_{i} \int_{0}^{\infty} G_{i}(s) u_{i}(t-s) d s-h_{i}(t)\right), \\
u_{i}^{\prime}(t)=-e_{i} u_{i}(t)+d_{i} x_{i}(t), \quad i=1,2, \ldots, n,
\end{array}\right.
$$

with initial conditions (1.2) satisfy $(x(t), u(t)) \rightarrow\left(x^{*}, u^{*}\right)$ as $t \rightarrow \infty$.

Proof. The result follows by repeating the above proofs with $H_{i}(t)$ in $(3.19),(4.5)$ or (4.9) replaced by $\mathcal{H}_{i}(t):=H_{i}(t)+h_{i}(t), i=1, \ldots, n$.

Example 4.1. We introduce a delayed control in the single population model proposed by Volterra and studied by Miller [17]:

$$
\left\{\begin{array}{l}
x^{\prime}(t)=x(t)\left(a-b x(t)-\int_{c}^{t} f(t-s) x(s) d s-\int_{c}^{t} g(t-s) u(s) d s\right), \\
u^{\prime}(t)=-e u(t)+d x(t),
\end{array}\right.
$$

where $c=0$ or $c=-\infty, a, b, d, e>0$, and the memory functions $f:[0, \infty) \rightarrow \mathbb{R}, g:[0, \infty) \rightarrow$ $[0, \infty)$ are continuous and in $L^{1}[0, \infty), g(0)>0$. For $c=-\infty,(4.11)$ is the autonomous system

$$
\left\{\begin{array}{l}
x^{\prime}(t)=x(t)\left(a-b x(t)-\int_{0}^{\infty} f(s) x(t-s) d s-\int_{0}^{\infty} g(s) u(t-s) d s\right), \\
u^{\prime}(t)=-e u(t)+d x(t)
\end{array}\right.
$$


whereas for $c=0(4.11)$ takes the form

$$
\left\{\begin{array}{l}
x^{\prime}(t)=x(t)\left(a-b x(t)-\int_{0}^{t} f(s) x(t-s) d s-\int_{0}^{t} g(s) u(t-s) d s\right), \\
u^{\prime}(t)=-e u(t)+d x(t) .
\end{array}\right.
$$

From Theorem 3.2 (see also Remark 3.3) and Corollary 4.1, if $b \geq(d / e) \int_{0}^{\infty} g(s) d s+\int_{0}^{\infty}|f(s)| d s$, then for any positive solution $(x(t), u(t))$ of (4.11) with either $c=0$ or $c=-\infty$, we have $x(t) \rightarrow$ $x^{*}=a\left[b+(d / e) \int_{0}^{\infty} g(s) d s+\int_{0}^{\infty} f(s) d s\right]^{-1}$ as $t \rightarrow \infty$.

When predator-prey systems (1.1) are considered, next result provides less restrictive sufficient conditions for the extinction of all the predator populations.

Theorem 4.4. Assume that $M$ is a P-matrix, let $\left(x^{*}, u^{*}\right)$ be the saturated equilibrium of (1.1), and suppose that $x^{*}=\left(x_{1}^{*}, \ldots, x_{p}^{*}, 0, \ldots, 0\right)(1 \leq p<n)$. With the notations of Theorem 4.2, assume that $A_{12} \geq 0, A_{21} \leq 0$. If $\hat{\mathcal{M}}_{11}, \mathcal{M}_{22}^{-}$are M-matrices, then $\left(x^{*}, u^{*}\right)$ is a global attractor for the solutions $(x(t), u(t))$ of (1.1)-(1.2).

Proof. Write $n_{1}=p, n_{2}=n-p$. Let $(x(t), u(t))$ be a positive solution of (1.1), and set $y_{i}(t)=x_{i}(t)-x_{i}^{*}, v_{i}(t)=u_{i}(t)-u_{i}^{*}$ for $1 \leq i \leq n_{1}$, and $y_{i}(t)=x_{i}(t), v_{i}(t)=u_{i}(t)$ for $n_{1}+1 \leq i \leq n$.

Claim 1. $\limsup _{t \rightarrow \infty} x_{i}(t) \leq x_{i}^{*}$ for $i=1, \ldots, n_{1}$.

With $A_{12} \geq 0$, together with equations (3.14) we get

$$
y_{i}^{\prime}(t) \leq-\left(y_{i}(t)+x_{i}^{*}\right)\left(\mu_{i} y_{i}(t)-\sum_{j=1}^{n_{1}}\left|a_{i j}\right| \int_{0}^{\infty} K_{i j}(s) y_{j}(t-s) d s+c_{i} \int_{0}^{\infty} G_{i}(s) v_{i}(t-s) d s\right)
$$

for $i=1,2, \ldots, n_{1}$. Fix any $\delta_{0}>0$ small. With $\hat{\mathcal{M}}_{11}$ an M-matrix, and after a scaling of the variables, we may suppose that $\left(\delta_{0} I_{n_{1}}+\hat{\mathcal{M}}_{11}\right) \eta>0$ for the positive vector $\eta=(1, \ldots, 1) \in \mathbb{R}^{n_{1}}$. Define $L_{i}=\lim \sup _{t \rightarrow \infty} y_{i}(t), U=\max _{1 \leq i \leq n_{1}} L_{i}$. We need to prove that $U \leq 0$.

Suppose that $U>0$. As for the estimates (3.20), for any $\varepsilon>0$ the definition of $U$ implies that $\int_{0}^{\infty} K_{i j}(s) y_{j}(t-s) d s \leq(U+\varepsilon)$ for $t>0$ large and $j=1, \ldots, n_{1}$. Applying the proof of Theorem 3.2 , it is clear that we shall get a contradiction, as in (3.22).

Claim 2. $\limsup _{t \rightarrow \infty} \int_{0}^{\infty} K_{i j}(s) y_{j}(t-s) d s \leq 0$ for $j=1, \ldots, n_{1}, i=1, \ldots, n$.

Fix $j \in\left\{1, \ldots, n_{1}\right\}, i \in\{1, \ldots, n\}$, and $\delta>0$. Since $y_{j}(t)$ is uniformly bounded in $\mathbb{R}$, there is $T_{1}>0$ such that $\int_{T_{1}}^{\infty} K_{i j}(s)\left|y_{j}(t-s)\right| d s \leq \delta / 2$. From Claim $1, \lim \sup _{t \rightarrow \infty} y_{j}(t) \leq 0$, hence there is $T_{2} \geq T_{1}$ such that $y_{j}(t)<\delta / 2$ for each $t \geq T_{2}$. Thus, for $t \geq 2 T_{2}$, we have

$$
\int_{0}^{\infty} K_{i j}(s) y_{j}(t-s) d s \leq \int_{0}^{T_{2}} K_{i j}(s) y_{j}(t-s) d s+\delta / 2<\delta .
$$

This proves Claim 2.

Claim 3. $\lim _{t \rightarrow \infty} x_{i}(t)=0$ for $i=n_{1}+1, \ldots, n$. 
For each $i \in\left\{n_{1}+1, \ldots, n\right\}$, we only need to prove that $\lim \sup _{t \rightarrow \infty} x_{i}(t) \leq 0$. Together with the equations $u_{i}^{\prime}(t)=-e_{i} x_{i}(t)+d_{i} u_{i}(t)$, we now obtain

$$
\begin{aligned}
x_{i}^{\prime}(t) & =x_{i}(t)\left(b_{i}-\mu_{i} x_{i}(t)-\sum_{j=1}^{n} a_{i j} \int_{0}^{\infty} K_{i j}(s) x_{j}(t-s) d s-c_{i} \int_{0}^{\infty} G_{i}(s) u_{i}(t-s) d s\right) \\
& \leq x_{i}(t)\left(\beta_{i}-\mu_{i} x_{i}(t)-\sum_{j=n_{1}+1}^{n} a_{i j} \int_{0}^{\infty} K_{i j}(s) x_{j}(t-s) d s-c_{i} \int_{0}^{\infty} G_{i}(s) u_{i}(t-s) d s-h_{i}(t)\right),
\end{aligned}
$$

where $\beta_{i}:=b_{i}-\sum_{j=1}^{n_{1}} a_{i j} x_{j}^{*} \leq 0$ (by the definition of a saturated equilibrium) and

$$
h_{i}(t)=\sum_{j=1}^{n_{1}} a_{i j} \int_{0}^{\infty} K_{i j}(s) y_{j}(t-s), \quad i=n_{1}+1, \ldots, n .
$$

From Claim 2 and since $A_{21} \leq 0$, we have $\lim \sup _{t \rightarrow \infty}\left(-h_{i}(t)\right) \leq 0$ for $i=n_{1}+1, \ldots, n$. From Corollary 4.1 (see also the proof of Theorem 4.2), the hypothesis $\mathcal{M}_{22}^{-}$is an M-matrix implies Claim 3.

Claim 4. $\lim _{t \rightarrow \infty} x_{i}(t)=x_{i}^{*}, \lim _{t \rightarrow \infty} u_{i}(t)=u_{i}^{*}$ for $i=1, \ldots, n_{1}$.

We write

$$
\left\{\begin{array}{c}
y_{i}^{\prime}(t)=-\left(y_{i}(t)+x_{i}^{*}\right)\left(\mu_{i} y_{i}(t)+\sum_{j=1}^{n_{1}} a_{i j} \int_{0}^{\infty} K_{i j}(s) y_{j}(t-s) d s\right. \\
\left.+c_{i} \int_{0}^{\infty} G_{i}(s) v_{i}(t-s) d s+h_{i}(t)\right), \\
v_{i}^{\prime}(t)=-e_{i} v_{i}(t)+d_{i} y_{i}(t), \quad i=1,2, \ldots, n_{1},
\end{array}\right.
$$

where now

$$
h_{i}(t)=\sum_{j=n_{1}+1}^{n} a_{i j} \int_{0}^{\infty} K_{i j}(s) x_{j}(t-s) d s, \quad i=1, \ldots, n_{1} .
$$

Using arguments as the ones above to prove Claim 2, where now we use Claim 3 instead of Claim 1 , we get $\lim _{t \rightarrow \infty} h_{i}(t)=0,1 \leq i \leq n_{1}$. Claim 4 follows again from Corollary 4.1.

It is straightforward to apply the above results to uncontrolled systems (3.1), which in the case of saturated equilibria on $\partial\left(\mathbb{R}_{+}^{n}\right)$ lead to better criteria than the ones in [4], as stated below.

Corollary 4.2. Assume that $M_{0}$ is a P-matrix, let $x^{*}$ be the saturated equilibrium of (3.1), and suppose that $x^{*}=\left(x_{1}^{*}, \ldots, x_{p}^{*}, 0, \ldots, 0\right)(1 \leq p<n)$. With the notations in the statement of Theorems 4.2, denote also

$$
\hat{\mathcal{M}}_{0}:=\left[\begin{array}{cc}
\hat{\mathcal{M}}_{0,11} & -\left|A_{12}\right| \\
-\mathcal{A}_{21} & \mathcal{M}_{0,22}^{-}
\end{array}\right]
$$

where

$$
\hat{\mathcal{M}}_{0,11}=\operatorname{diag}\left(\mu_{1}, \ldots, \mu_{p}\right)-\left|A_{11}\right|, \quad \mathcal{M}_{0,22}^{-}=\operatorname{diag}\left(\mu_{p+1}, \ldots, \mu_{n}\right)-A_{22}^{-}
$$

and $\mathcal{A}_{21}=\left[\tilde{a}_{i j}\right]$ is given by (4.7). If $\hat{\mathcal{M}}_{0}$ is a non-singular $M$-matrix, then $x^{*}$ is a global attractor for all positive solutions $x(t)$ of (3.1). 
Moreover, if either (i) $A_{21} \geq 0, b_{i} \leq 0$ for $p<i \leq n$, or (ii) $A_{12} \geq 0, A_{21} \leq 0$, and $\hat{\mathcal{M}}_{0,11}, \mathcal{M}_{0,22}^{-}$ are non-singular M-matrices, then $x^{*}$ is a global attractor for the positive solutions of (3.1).

\section{The two-species Lotka-Volterra system}

As an application of the results in the previous sections, we now analyse with some attention the dynamics for a planar controlled Lotka-Volterra system with delays, without any special constraints on the signs of the Malthusian coefficients $b_{i}$ and intra- and inter-specific coefficients $a_{i j}$. For the sake of simplicity, we consider a planar system (1.1) with discrete delays, but the analysis below can be performed for infinite distributed delays as well.

Consider the system

$$
\left\{\begin{array}{l}
x_{1}^{\prime}(t)=x_{1}(t)\left(b_{1}-\mu_{1} x_{1}(t)-a_{11} x_{1}\left(t-\tau_{11}\right)-a_{12} x_{2}\left(t-\tau_{12}\right)-c_{1}^{0} u_{1}(t)-c_{1}^{1} u_{1}\left(t-\sigma_{1}\right)\right) \\
u_{1}^{\prime}(t)=-e_{1} u_{1}(t)+d_{1} x_{1}(t) \\
x_{2}^{\prime}(t)=x_{2}(t)\left(b_{2}-\mu_{2} x_{2}(t)-a_{21} x_{1}\left(t-\tau_{21}\right)-a_{22} x_{2}\left(t-\tau_{22}\right)-c_{2}^{0} u_{2}(t)-c_{2}^{1} u_{2}\left(t-\sigma_{2}\right)\right) \\
u_{2}^{\prime}(t)=-e_{2} u_{2}(t)+d_{2} x_{2}(t)
\end{array}\right.
$$

where: $\mu_{i}, c_{i}^{0}, d_{i}, e_{i}$ are positive constants, $c_{i}^{1} \geq 0, b_{i}, a_{i j} \in \mathbb{R}, \tau_{i j}, \sigma_{i} \geq 0, i, j=1,2$. Denote $c_{i}=c_{i}^{0}+c_{i}^{1}, i=1,2$. With the above notation, the community matrix is

$$
M=\left[\begin{array}{cc}
\lambda_{1}+a_{11} & a_{12} \\
a_{21} & \lambda_{2}+a_{22}
\end{array}\right] \text { where } \quad \lambda_{i}=\mu_{i}+\frac{c_{i} d_{i}}{e_{i}}, i=1,2 .
$$

In what follows, we suppose in addition that $M$ is P-matrix, i.e.,

$$
\operatorname{det} M>0 \quad \text { and } \quad \lambda_{i}+a_{i i}>0, i=1,2 \text {. }
$$

There are three possible equilibria on the boundary of $\mathbb{R}_{+}^{4}$ : the trivial equilibrium $E_{0}=$ $(0,0,0,0), E_{1}=\left(\frac{b_{1}}{\lambda_{1}+a_{11}}, \frac{b_{1} d_{1}}{\left(\lambda_{1}+a_{11}\right) e_{1}}, 0,0\right)$ if $b_{1}>0$, and $E_{2}=\left(0,0, \frac{b_{2}}{\lambda_{2}+a_{22}}, \frac{b_{2} d_{2}}{\left(\lambda_{2}+a_{22}\right) e_{2}}\right)$ if $b_{2}>0$. There is a positive equilibrium $E^{*}=\left(x_{1}^{*}, u_{1}^{*}, x_{2}^{*}, u_{2}^{*}\right)$, where

$$
x_{1}^{*}=\frac{b_{1}\left(\lambda_{2}+a_{22}\right)-a_{12} b_{2}}{\operatorname{det} M}, \quad x_{2}^{*}=\frac{b_{2}\left(\lambda_{1}+a_{11}\right)-a_{21} b_{1}}{\operatorname{det} M}, \quad u_{i}^{*}=\frac{d_{i}}{e_{1}} x_{i}^{*}, i=1,2,
$$

if and only if

$$
b_{1}\left(\lambda_{2}+a_{22}\right)>a_{12} b_{2}, \quad b_{2}\left(\lambda_{1}+a_{11}\right)>a_{21} b_{1} .
$$

As already observed, the trivial equilibrium is saturated if and only if $b_{1}, b_{2} \leq 0$. In this case, 0 is globally attractive if $\mu_{i}-a_{i i}^{-} \geq 0(i=1,2)$ and $\left(\mu_{1}-a_{11}^{-}\right)\left(\mu_{2}-a_{22}^{-}\right) \geq a_{12}^{-} a_{21}^{-}$. If $b_{i}>0$, then $E_{i}$ is an equilibrium on the boundary of the positive cone, $i=1,2$.

Next, we give a detailed analysis of the absolute stability, and lack of it, for the case of $b_{1}, b_{2}$ positive. The case $b_{2} \leq 0<b_{1}$ will be studied afterwards.

Let $b_{1}, b_{2}>0$, so that the equilibria $E_{0}, E_{1}, E_{2}$ always exist, with $E_{0}$ unstable. At least one of the conditions in (5.3) is satisfied; otherwise, we get $a_{12} b_{2} \geq b_{1}\left(\lambda_{2}+a_{22}\right)>0$ and $a_{21} b_{1} \geq$ $b_{2}\left(\lambda_{1}+a_{11}\right)>0$ and $\left[\left(\lambda_{1}+a_{11}\right)\left(\lambda_{2}+a_{22}\right)-a_{12} a_{21}\right] b_{1} b_{2}=b_{1} b_{2} \operatorname{det} M \leq 0$, which is not possible. 
We now study the stability of $E_{1}$. Clearly, a similar analysis can be performed for $E_{2}$. The characteristic equation for the linearised equation about $E_{1}=\left(X_{1}, \frac{d_{1}}{e_{1}} X_{1}, 0,0\right)$ is given by

$$
\operatorname{det} \Delta(\lambda)=0 \text { for } \Delta(\lambda)=\lambda I_{4}+\left[\begin{array}{cc}
N(\lambda) & E(\lambda) \\
0 & C
\end{array}\right]
$$

( $I_{n}$ is the $n \times n$ identity matrix), where $X_{1}=\frac{b_{1}}{\lambda_{1}+a_{11}}$ and

$$
\begin{gathered}
N(\lambda)=\left[\begin{array}{cc}
X_{1}\left(\mu_{1}+a_{11} e^{-\lambda \tau_{11}}\right) & X_{1}\left(c_{1}^{0}+c_{1}^{1} e^{-\lambda \sigma_{1}}\right) \\
-d_{1} & e_{1}
\end{array}\right], \quad E(\lambda)=\left[\begin{array}{cc}
X_{1} a_{12} e^{-\lambda \tau_{12}} & 0 \\
0 & 0
\end{array}\right], \\
C=\left[\begin{array}{cc}
-\left(b_{2}-a_{21} X_{1}\right) & 0 \\
-d_{2} & e_{2}
\end{array}\right] .
\end{gathered}
$$

If $b_{2}\left(\lambda_{1}+a_{11}\right)>a_{21} b_{1}$, then $b_{2}-a_{21} X_{1}>0$, and $E_{1}$ is unstable. If $b_{2}\left(\lambda_{1}+a_{11}\right) \leq a_{21} b_{1}$, the matrix $-C$ is stable, therefore $E_{1}$ is the unique saturated equilibrium. In fact, in this situation, there is no positive equilibrium, but, as already observed, the condition $b_{1}\left(\lambda_{2}+a_{22}\right)>a_{12} b_{2}$ must hold, and from a dual analysis we would conclude that $E_{2}$ is unstable.

When $E_{1}$ is the unique saturated equilibrium, conditions (5.2) are not however sufficient to conclude that $E_{1}$ is a global attractor of all positive solutions for all sizes of the delays $\tau_{11}, \sigma_{1}$. In fact, the characteristic roots of (5.4) are $\lambda=-e_{2}<0, \lambda=b_{2}-a_{21} X_{1} \leq 0$ and the solutions of $h(\lambda)=0$, where

$$
h(\lambda)=P(\lambda)+e^{-\lambda \tau_{11}} Q(\lambda)+X_{1} d_{1} c_{1}^{1} e^{-\lambda \sigma_{1}}
$$

with $P(\lambda)=\lambda^{2}+\lambda\left(e_{1}+X_{1} \mu_{1}\right)+X_{1}\left(\mu_{1} e_{1}+d_{1} c_{1}^{0}\right), Q(\lambda)=a_{11} X_{1}\left(\lambda+e_{1}\right)$. The equation $h(\lambda)=0$ is the characteristic equation for the system

$$
\left\{\begin{array}{l}
x^{\prime}(t)=-X_{1}\left[\mu_{1} x(t)+a_{11} x\left(t-\tau_{11}\right)+c_{1}^{0} u(t)+c_{1}^{1} u\left(t-\sigma_{1}\right)\right] \\
u^{\prime}(t)=-\left[e_{1} u(t)-d_{1} x(t)\right] .
\end{array}\right.
$$

With $\tau_{11}, \sigma_{1}=0$, the solutions $\lambda$ of $h(\lambda)$ are the eigenvalues of the matrix

$$
-N(0)=-\left[\begin{array}{cc}
X_{1}\left(\mu_{1}+a_{11}\right) & X_{1} c_{1} \\
-d_{1} & e_{1}
\end{array}\right],
$$

which has $\operatorname{det}(-N(0))=X_{1}\left(\lambda_{1}+a_{11}\right) e_{1}>0($ from $(5.2))$ and trace $T_{0}:=-X_{1}\left(\mu_{1}+a_{11}\right)-e_{1}$. If $T_{0} \leq 0$, then $E_{1}$ is stable as an equilibrium of system (5.6) with $\tau_{11}, \sigma_{1}=0$, otherwise, $E_{1}$ is unstable. It is particularly difficult to study a second order characteristic equation with two delays, as equation (5.5), cf. e.g. [2, 25] and references therein. For instance, fixing $\sigma_{1}=0$, if $T_{0}<0$, in general there is some $\tau^{*} \geq 0$ such that the above system (5.6) is stable for delays $\tau_{11}<\tau^{*}$, and unstable if $\tau_{11}>\tau^{*}$, or the stability can change a finite number of times as $\tau_{11}$ increases, and eventually it becomes unstable - and therefore, although saturated, $E_{1}$ becomes unstable also for (5.1). Now assume that $\mu_{1}-\left|a_{11}\right|-c_{1} \frac{d_{1}}{e_{1}} \geq 0$. Then, the trace $T_{0}$ of $-N(0)$ is always negative, hence $E_{1}$ is asymptotically stable for system (5.6) with $\tau_{11}, \sigma_{1}=0$. Moreover, the matrix

$$
\hat{N}(0)=\left[\begin{array}{cc}
X_{1}\left(\mu_{1}-\left|a_{11}\right|\right) & -X_{1} c_{1} \\
-d_{1} & e_{1}
\end{array}\right]
$$


$\operatorname{has} \operatorname{det} \hat{N}(0)=X_{1} e_{1}\left(\mu_{1}-\left|a_{11}\right|-c_{1} \frac{d_{1}}{e_{1}}\right) \geq 0$ and trace $\hat{T}_{0}=X_{1}\left(\mu_{1}-\left|a_{11}\right|\right)+e_{1}>0$, hence $\hat{N}(0)$ is an M-matrix [7]. By [5], it follows that system (5.6) is exponentially stable for all delays $\tau_{11}, \sigma_{1}>0$. By Theorem 4.2, $E_{1}$ is the global attractor of all positive solutions of (5.1) if

$$
\hat{\mathcal{M}}=\left[\begin{array}{cc}
\mu_{1}-\left|a_{11}\right|-c_{1} \frac{d_{1}}{e_{1}} & -\left|a_{12}\right| \\
-a_{21} & \mu_{2}-a_{22}^{-}
\end{array}\right]
$$

is an M-matrix, or, in other words

$$
\mu_{1}-\left|a_{11}\right|-c_{1} \frac{d_{1}}{e_{1}} \geq 0, \mu_{2}-a_{22}^{-} \geq 0,\left(\mu_{1}-\left|a_{11}\right|-c_{1} \frac{d_{1}}{e_{1}}\right)\left(\mu_{2}-a_{22}^{-}\right) \geq\left|a_{12}\right| a_{21} .
$$

Assume now (5.3), so that the positive equilibrium $E^{*}$ exists. For the linearised equation about $E^{*}$, written as

$$
X^{\prime}(t)=-\left[D X(t)+L\left(X_{t}\right)\right]
$$

where $D=\operatorname{diag}\left(x_{1}^{*} \mu_{1}, e_{1}, x_{2}^{*} \mu_{2}, e_{2}\right)$, the characteristic equation is given by $\operatorname{det} \Delta(\lambda):=\lambda I_{4}+D+$ $L\left(e^{\lambda \cdot} I_{4}\right)=0$, and similar computations as the ones above lead to

$$
D+L\left(e^{\lambda \cdot} I_{4}\right)=\left[\begin{array}{ll}
N_{1}(\lambda) & E_{1}(\lambda) \\
E_{2}(\lambda) & N_{2}(\lambda)
\end{array}\right]
$$

where

$$
N_{i}(\lambda)=\left[\begin{array}{cc}
x_{i}^{*}\left(\mu_{i}+a_{i i} e^{-\lambda \tau_{i i}}\right) & x_{i}^{*}\left(c_{i}^{0}+c_{i}^{1} e^{-\lambda \sigma_{i}}\right) \\
-d_{i} & e_{i}
\end{array}\right], E_{i}(\lambda)=\left[\begin{array}{cc}
x_{i}^{*} a_{i j} e^{-\lambda \tau_{i j}} & 0 \\
0 & 0
\end{array}\right], \quad i, j=1,2, j \neq i .
$$

One can easily check that $\operatorname{det} \Delta(0)=\operatorname{det}\left(D+L\left(I_{4}\right)\right)=x_{1}^{*} x_{2}^{*} e_{1} e_{2} \operatorname{det} M$, thus $\operatorname{det} \Delta(0)>0$ since $M$ is a P-matrix. As for the study of the stability of $E_{1}$, even if $E^{*}$ is asymptotically stable for the corresponding ODE system obtained by taking all the delays equal to zero in (5.1), the positive equilibrium $E^{*}$ of (5.1) might become unstable as the delays increase. In fact, by letting $c_{1}, c_{2} \rightarrow 0^{+}$, from (5.8) we obtain $\operatorname{det} \Delta(\lambda) \rightarrow\left(\lambda+e_{1}\right)\left(\lambda+e_{2}\right) h(\lambda)$, where now

$$
h(\lambda)=\left|\begin{array}{cc}
\lambda+x_{1}^{*}\left(\mu_{1}+a_{11} e^{-\lambda \tau_{11}}\right) & x_{1}^{*} a_{12} e^{-\lambda \tau_{12}} \\
x_{2}^{*} a_{21} e^{-\lambda \tau_{21}} & \lambda+x_{2}^{*}\left(\mu_{2}+a_{22} e^{-\lambda \tau_{22}}\right)
\end{array}\right| .
$$

Choosing e.g. $\tau_{i i}=0,(i=1,2)$ and $a_{12}=1, a_{21}=-1$, one can see that it is possible to choose the other coefficients in such a way that $\left(\mu_{1}+a_{11}\right) x_{1}^{*}=\left(\mu_{2}+a_{22}\right) x_{2}^{*}=: b$ and $x_{1}^{*} x_{2}^{*}=: c>b^{2}$. Then, $h(\lambda)=(\lambda+b)^{2}+c e^{-\lambda\left(\tau_{12}+\tau_{21}\right)}$, which has roots $\pm i \sqrt{c-b^{2}}$ if $\tau:=\tau_{12}+\tau_{21}=\tau_{n}$, where

$$
\tau_{n} \in(0, \pi)+2 n \pi, \quad \tan \left(\tau_{n} \sqrt{c-b^{2}}\right)=\frac{2 b \sqrt{c-b^{2}}}{c-2 b^{2}}, \quad n=0,1,2, \ldots
$$

In particular, for $\tau>\tau_{0}$ and close to $\tau_{0}$, there is a pair of characteristic roots with positive real parts, thus the equilibrium becomes unstable. Moreover, system (5.1) has a sequence of Hopf bifurcations at $\tau=\tau_{n}, n=0,1,2, \ldots[23]$. On reverse, if

$$
\hat{M}=\left[\begin{array}{cc}
\mu_{1}-\left|a_{11}\right|-c_{1} \frac{d_{1}}{e_{1}} & -\left|a_{12}\right| \\
-\left|a_{21}\right| & \mu_{2}-\left|a_{22}\right|-c_{2} \frac{d_{2}}{e_{2}}
\end{array}\right]
$$


is an M-matrix, so that we have the conditions

$$
\left(\mu_{1}-\left|a_{11}\right|-c_{1} \frac{d_{1}}{e_{1}}\right)\left(\mu_{2}-\left|a_{22}\right|-c_{2} \frac{d_{2}}{e_{2}}\right) \geq\left|a_{12} a_{21}\right| \text { and } \mu_{i}-\left|a_{i i}\right|-c_{i} \frac{d_{i}}{e_{i}} \geq 0, i=1,2,
$$

the positive equilibrium $E^{*}$ is globally attractive, for all sizes of delays $\tau_{i j}, \sigma_{i}$.

As an application of the use of the controls, in the example below we change the position of the globally attractive equilibrium, from the boundary to the interior of $\mathbb{R}_{+}^{2}$, recovering one of the species, otherwise condemned to extinction.

Example 5.1. Consider the following uncontrolled system with $n=2$ and e.g. $b_{1}=1, b_{2}=\frac{1}{3}, \mu_{1}=$ $\mu_{2}=1, a_{11}=a_{22}=a_{21}=\frac{1}{2}, a_{12}=\frac{1}{8}$ :

$$
\left\{\begin{array}{l}
x_{1}^{\prime}(t)=x_{1}(t)\left(1-x_{1}(t)-\frac{1}{2} x_{1}\left(t-\tau_{11}\right)-\frac{1}{8} x_{2}\left(t-\tau_{12}\right)\right) \\
x_{2}^{\prime}(t)=x_{2}(t)\left(\frac{1}{3}-x_{2}(t)-\frac{1}{2} x_{1}\left(t-\tau_{21}\right)-\frac{1}{2} x_{2}\left(t-\tau_{22}\right)\right)
\end{array} .\right.
$$

With the above notations, we have $M_{0}=\left[\begin{array}{ll}3 / 2 & 1 / 8 \\ 1 / 2 & 3 / 2\end{array}\right], \hat{M}_{0}=\left[\begin{array}{cc}1 / 2 & -1 / 8 \\ -1 / 2 & 1 / 2\end{array}\right]$. Its saturated equilibrium is $\left(X_{1}, 0\right)=\left(\frac{2}{3}, 0\right)$. Furthermore, $\operatorname{det} M_{0}>0$ and $\hat{M}_{0}$ is a non-singular M-matrix, hence from [4] we derive that $\left(X_{1}, 0\right)$ is GAS. We now introduce the controls, in order to recover the $x_{2}(t)$ population, which otherwise would become extinct with time. Clearly, for any choice of positive coefficients $c_{i}, d_{i}, e_{i}, i=1,2$, conditions (5.3) hold, and therefore the controlled system (5.1) with the above coefficients has a positive equilibrium $E^{*}$. Now, if we choose e.g. $\alpha_{i}:=c_{i} \frac{d_{i}}{e_{i}} \leq \frac{1}{4}, i=1,2$, we have that

$$
\hat{M}=\left[\begin{array}{cc}
1 / 2-\alpha_{1} & -1 / 8 \\
-1 / 2 & 1 / 2-\alpha_{2}
\end{array}\right]
$$

is an M-matrix. Invoking Theorem 3.2, we get that $E^{*}$ is a global attractor of all positive solutions.

Now, suppose that $b_{2} \leq 0<b_{1}$ and $a_{21} \geq 0$. Clearly (5.3) fails to be true, $E_{1}$ is the saturated equilibrium, and by Theorem $4.3 E_{1}$ is a global attractor of all positive solutions of (5.1) if

$$
\mu_{1}-\left|a_{11}\right|-c_{1} \frac{d_{1}}{e_{1}} \geq 0, \quad \mu_{2}-a_{22}^{-} \geq 0
$$

Next, consider a typical predator-prey system (5.1), where $b_{2}<0<b_{1}$ and $a_{12}>0, a_{21}<0$. In the absence of the positive equilibrium, which amounts to have $b_{2}\left(\lambda_{1}+a_{11}\right) \leq a_{21} b_{1}$, then $E_{1}$ is the saturated equilibrium. Using now Theorem 4.4, if (5.10) is satisfied, then again $E_{1}$ is a global attractor. In this framework, we again illustrate how the controls can be used to change the position of a globally attractive saturated equilibrium.

Example 5.2. For the particular case of $b_{1}=1, b_{2}=-\frac{5}{4}, \mu_{1}=\mu_{2}=1, a_{11}=a_{22}=\frac{1}{2}, a_{12}=$ $\frac{1}{8}, a_{21}=-2$, we obtain the predator-prey system without controls

$$
\left\{\begin{array}{l}
x_{1}^{\prime}(t)=x_{1}(t)\left(1-x_{1}(t)-\frac{1}{2} x_{1}\left(t-\tau_{11}\right)-\frac{1}{8} x_{2}\left(t-\tau_{12}\right)\right) \\
x_{2}^{\prime}(t)=x_{2}(t)\left(-\frac{5}{4}-x_{2}(t)+2 x_{1}\left(t-\tau_{21}\right)-\frac{1}{2} x_{2}\left(t-\tau_{22}\right)\right)
\end{array},\right.
$$


with community matrix $M_{0}=\left[\begin{array}{cc}3 / 2 & 1 / 8 \\ -2 & 3 / 2\end{array}\right]$. For this system, $\left(x_{1}^{*}, x_{2}^{*}\right)=\left(\frac{53}{80}, \frac{1}{20}\right)$ is the positive equilibrium. Moreover, since $\operatorname{det} M_{0}>0$ and $\hat{M}_{0}=\left[\begin{array}{cc}1 / 2 & -1 / 8 \\ -2 & 1 / 2\end{array}\right]$ is an M-matrix, from [4] it follows that $\left(x_{1}^{*}, x_{2}^{*}\right)$ is globally atractive. We now introduce the controls, in order to drive the predators to extinction. For the above chosen coefficients, $b_{2}\left(\mu_{1}+a_{11}+c_{1} \frac{d_{1}}{e_{1}}\right) \leq a_{21} b_{1}$ if and only if $c_{1} \frac{d_{1}}{e_{1}} \geq \frac{1}{10}$, in which case $E_{1}=\left(\frac{1}{\frac{3}{2}+c_{1} \frac{d_{1}}{e_{1}}}, 0, \frac{d_{1} e_{1}}{\frac{3}{2} e_{1}+c_{1} d_{1}}, 0\right)$ is the saturated equilibrium. If we now choose $\frac{1}{10} \leq c_{1} \frac{d_{1}}{e_{1}} \leq \frac{1}{2}$, Theorem 4.4 yields that $\mu_{1}-\left|a_{11}\right|-c_{1} \frac{d_{1}}{e_{1}} \geq 0$, thus $E_{1}$ is a global attractor of all positive solutions.

We summarise the above global asymptotic behaviour results as follows:

Proposition 5.1. Consider system (5.1), and assume (5.2).

(i) If $b_{1}, b_{2} \leq 0$, then 0 is the saturated equilibrium; in this case, 0 is globally attractive if $\mu_{i}-a_{i i}^{-} \geq 0(i=1,2)$ and $\left(\mu_{1}-a_{11}^{-}\right)\left(\mu_{2}-a_{22}^{-}\right) \geq a_{12}^{-} a_{21}^{-}$.

(ii) If (5.3) holds, there exist a positive equilibrium, which is GAS under the additional conditions (5.9).

(iii) If $b_{1}, b_{2}>0$ and $b_{2}\left(\lambda_{1}+a_{11}\right) \leq a_{21} b_{1}$, then $E_{1}$ is the saturated equilibrium; in this case, $E_{1}$ is a global attractor of all positive solutions if conditions (5.7) are satisfied.

(iv) If $b_{2} \leq 0<b_{1}$, and: (a) either $a_{21} \geq 0$, or (b) $a_{12}>0, a_{21}<0, b_{2}\left(\lambda_{1}+a_{11}\right) \leq a_{21} b_{1}$, then $E_{1}$ is the saturated equilibrium; in this case, $E_{1}$ is a global attractor of all positive solutions if conditions (5.10) are satisfied.

\section{References}

1. A. Berman and R. Plemmons. Nonnegative Matrices in the Mathematical Sciences(Academic Press, New York, 1979).

2. S.A. Campbell and Y. Yuan. Zero singularities of codimension two and three in delay differential equations. Nonlinearity 21 (2008), 2671-2691.

3. F. Chen. The permanence and global attractivity of Lotka-Volterra competition system with feedback controls. Nonlinear Anal. RWA 7 (2006), 133-143.

4. T. Faria. Stability and Extinction for Lotka-Volterra Systems with Infinite Delay. J. Dynam. Differential Equations 22 (2010), 299-324.

5. T. Faria and J.J. Oliveira. Local and global stability for Lotka-Volterra systems with distributed delays and instantaneous feedbacks. J. Differential Equations 244 (2008), 1049-1079.

6. T. Faria and J.J. Oliveira. General criteria for asymptotic and exponencial stabilities of neural network models with unbounded delay. Appl. Math. Comput. 217 (2011), 9646-9658.

7. M. Fiedler. Special Matrices and Their Applications in Numerical Mathematics (Martinus Nijhoff Publ., Kluwer, Dordrecht, 1986).

8. K. Gopalsamy and P. Weng. Global attractivity in a competition system with feedback controls. Comput. Math. Appl. 45 (2003), 665-676.

9. J. Haddock and W. Hornor. Precompactness and convergence in norm of positive orbits in a certain fading memory space. Funkcial. Ekvac. 31 (1988), 349-361. 
10. J. Haddock, M.N. Nkashama and J.H. Wu. Asymptotic constancy for linear neutral Volterra integrodifferential equations. Tohoku Math. J. 41 (1989), 689-710.

11. J.K. Hale. Asymptotic Behavior of Dissipative Systems (Amer. Math. Soc., Providence, 1988).

12. J.K. Hale and J. Kato. Phase space for retarded equations with infinite delay. Funkcial. Ekvac. 21 (1978), 11-41.

13. Y. Hino, S. Murakami and T. Naito. Functional Differential Equations with Infinite Delay (Springer-Verlag, New-York, 1993).

14. J. Hofbauer and K. Sigmund. The Theory of Evolution and Dynamical Systems (London Mathematical Society, Cambridge University Press, Cambridge, 1988).

15. Y. Kuang. Global stability in delay differential systems without dominating instantaneous negative feedbacks. J. Differential Equations 119 (1995), 503-532.

16. Z. Li, M. Han and F. Chen. Influence of feedback controls on an autonomous Litoka-Volterra competitive system with infinite delays. Nonlinear Anal. RWA 14 (2013), 402-413.

17. R.K. Miller. On Volterra's population equation. SIAM J. Appl. Math. 14 (1966), 446-452.

18. F. Montes de Oca and M. Vivas. Extinction in a two dimensional Lotka-Volterra system with infinite delay. Nonlinear Anal. RWA 7 (2006), 1042-1047.

19. Y. Muroya. Partial survival and extinction of species in nonutonomous Lotka-Volterra systems with delays. Dynamic Systems and Applications 12 (2003), 295-306.

20. L. Nie, J. Peng and Z. Teng. Permanence and stability in multi-species non-autonomous LotkaVolterra competitive systems with delays and feedback controls. Math. Comput. Modelling 49 (2009), 295-306.

21. C. Shi, Z. Li and F. Chen. Extinction in a nonautonomous Lotka-Volterra competitive system with infinite delay and feedback controls. Nonlinear Anal. RWA 13 (2012), 2214-2226.

22. H.L. Smith. Monotone Dynamical Systems. An Introduction to the Theory of Competitive and Cooperative Systems (Amer. Math. Soc., Providence, 1995).

23. P. Táboas. Periodic solutions of a planar delay equation. Proc. Roy. Soc. Edinburgh Sect. A 116 (1990), 85-101.

24. P. Weng. Existence and global stability of positive periodic solution of periodic integrodifferential systems with feedback controls. Comput. Math. Appl. 40 (2000), 747-759.

25. D. Xiao and S. Ruan. Multiple bifurcations in a delayed predator-prey system with nonmonotonic functional response. J. Differential Equations 176 (2001), 494-510.

26. R. Xu, M.A.J. Chaplain and F.A. Davidson. Global asymptotic stability in a nonautonomous $n$-species Lotka-Volterra predator-prey system with infinite delays. Appl. Anal. 80(2002), $107-126$.

27. Z. Zhang. Existence and global attractivity of a positive periodic solution for a generalized delayed population model with stocking and feedback control. Math. Comput. Modelling 48 (2008), 749-760. 